\title{
Steroidal Saponins from the Genus Smilax and Their Biological Activities
}

\author{
Li-Wen Tian (I) Zhen Zhang • Hai-Lan Long • \\ Ying-Jun Zhang
}

Received: 16 April 2017/Accepted: 12 June 2017/Published online: 23 June 2017

(c) The Author(s) 2017. This article is an open access publication

\begin{abstract}
The Smilax species, widely distributed in tropical region of the world and the warm areas of East Asia and North America, are extensively used as folk medicine to treat inflammatory disorders. Chemical investigation on Smilax species showed they are rich sources of steroidal saponins with diversified structure types, including spirostane, isospirostane, furostane, pregnane, and cholestane. This review mainly summarizes the steroidal saponins (1-104) reported from the genus Smilax between 1967 and 2016, and their biological activities. The relationship between structures of steroidal saponins and related biological activities were briefly discussed.
\end{abstract}

L.-W. Tian $(\bowtie) \cdot$ Z. Zhang $\cdot$ H.-L. Long

School of Pharmaceutical Sciences, Southern Medical

University, Guangzhou 510515, China

e-mail: 1wtian@smu.edu.cn

\section{Y.-J. Zhang}

State Key Laboratory of Phytochemistry and Plant Resources in West China, Kunming Institute of Botany, Chinese Academy of Sciences, Kunming 650201, China 


\section{Graphical Abstract}

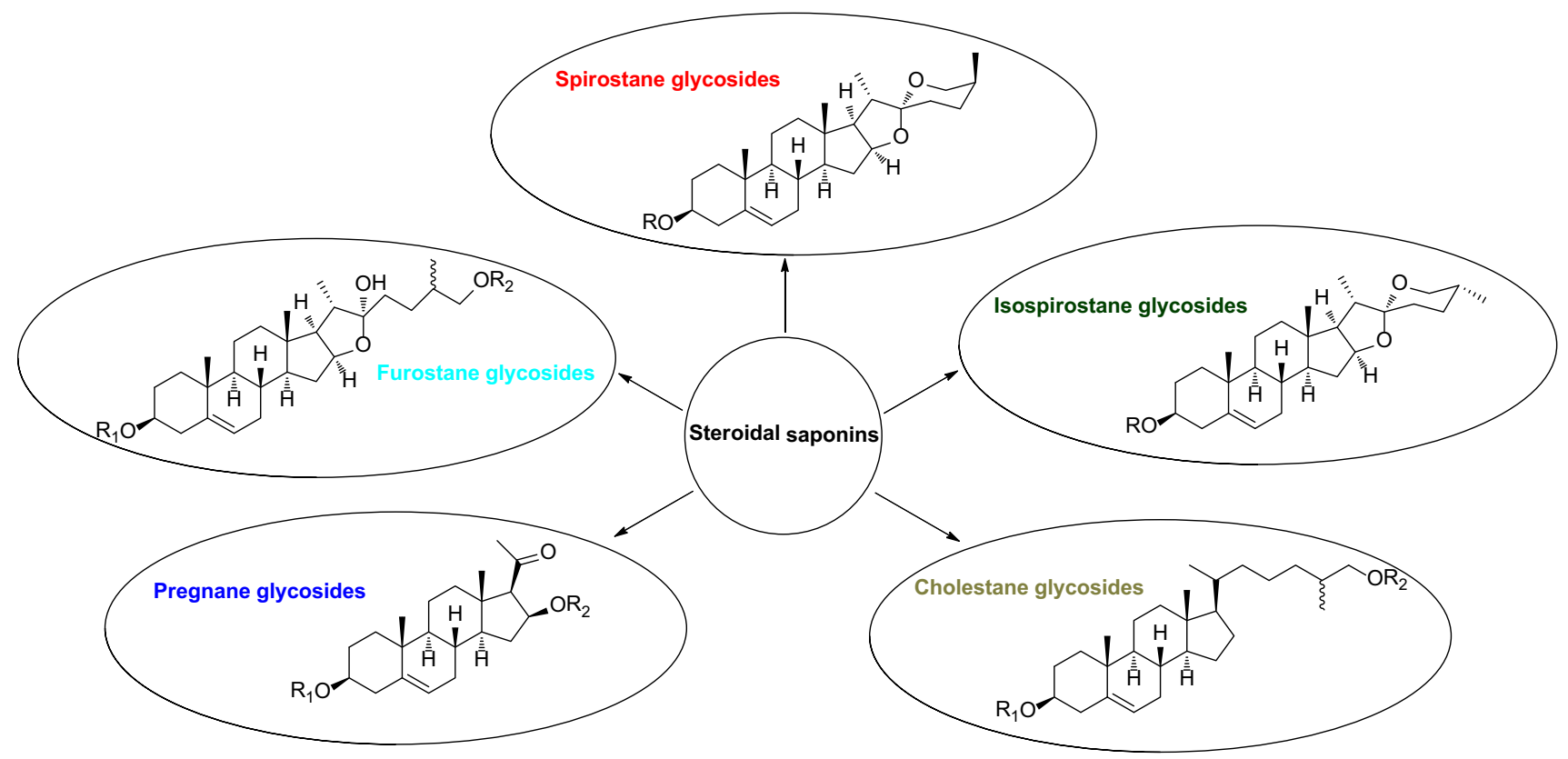

Keywords Smilax $\cdot$ Steroidal saponins $\cdot$ Biological activities

\section{Introduction}

The genus Smilax (Liliaceae family) comprises about 300 species of climbing shrubs. Plants of the genus are widely distributed in tropical region of the world, and also found in warm areas of East Asia and North America [1]. The juvenile leaves of S. riparia are used as vegetable product. The rhizomes of S. glabra are used in Southeast of China as food supplementary for health. Noteworthily, the rhizomes of Smilax species are most famous for their medical use. The rhizomes of S. china and S. glabra, called "Jin Gang Teng" and "Tu Fu Lin" in Pharmacopoeia of People's Republic of China respectively, are clinically used to treat chronic pelvic inflammatory disease, rheumatic arthritis and so on [2]. The rhizomes of S. riparia, S. nipponica, $S$. bockii, S. microphylla, and S. discotis were recorded in the Chinese Herbal Medicines to treat joint pain, edema, and rheumatoid arthritis [3].

Previous studies on chemical constituents of Smilax species have disclosed the presence of steroidal saponins, flavonoids, phenylpropanoids, and stilbenoids [4]. Astilbin, a main flavonoid among Smilax species [5], showed unique immunosuppressive activity, and proved to be the active material basis of Smilax species for the treatment of human immune diseases [6]. Steroidal saponins are characteristic bioactive components of the genus Smilax in terms of chemotaxonomic value and biological activities [7]. So far, 104 steroidal saponins have been reported from 20 different Smilax species. These steroidal saponins showed significant antifungal, cytotoxic, anti-inflammatory, as well as cAMP phosphodiesterase inhibitory activities.

In this review, steroidal saponins reported from the genus Smilax between 1967 and 2016 were listed, and the biological activities of steroidal saponins were also included.

\section{Chemistry of Steroidal Saponins}

Steroidal saponins from the genus Smilax could be divided into five groups on the basis of the sapogenin structures: spirostane (A), isospirostane (B), furostane (C), pregnane (D), and cholestane (E) (Fig. 1). They are mostly mono- or bisdesmosides. A carbohydrate chain is always attached to the C-3 position of sapogenin by an ether linkage. Additionally, C-26 position of furostane-type saponin is always etherified with a glucopyranosyl moiety. So far only one steroidal saponin from the genus Smilax, (25S)-26- $O-\beta$-D- 


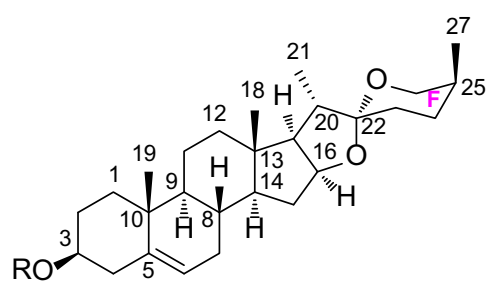

A

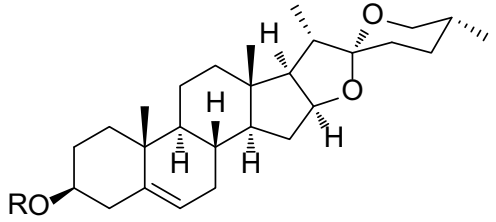

B

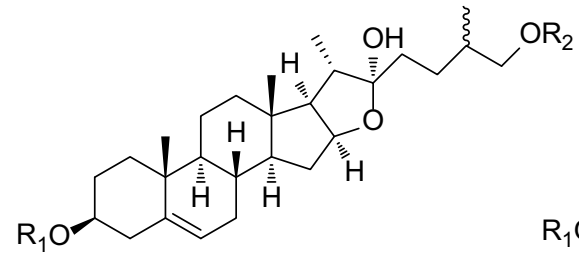

C

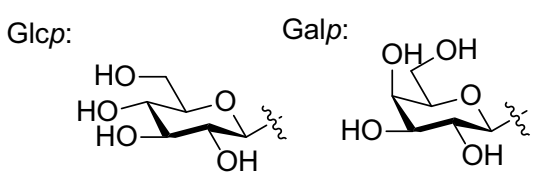

Rhap:

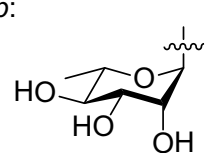

Fruf:<smiles>OCC1O[C@@H](O)C(O)C1O</smiles>

Arap:<smiles>OC1OC2CCCC(O2)C1O</smiles>

Fig. 1 Structures of a a spirostane backbone, $\mathbf{b}$ an isospirostane backbone, $\mathbf{c}$ a furostane backbone, $\mathbf{d}$ a pregnane backbone, e a cholestane backone, a glucopyranosyl moiety (Glcp), a

glucopyranosyl-5 $\beta$-furostan-1 $\beta, 3 \beta, 22 \alpha, 26$-tetraol-1- $O-\beta$-Dglucopyranoside (92), has a glucopyranosyl moiety linked to the $\mathrm{C}-1$ position. The sugar residues consist of linear or branched saccharidic chains, made up most often of glucopyranosyl (Glcp), rhamnopyranosyl (Rhap), galactopyranosyl (Galp), fructofuronosyl (Fruf), and arabinopyranosyl (Arap) moieties (Fig. 1).

\subsection{Spirostane-Type Saponins 1-11}

Spirostane-type saponins are monodesmosidic glycosides with six rings A-F in sapogenin. They are characterized by an axial oriented methyl or hydroxymethyl (C-27) on F ring. The sapogenin of spirostane glycosides 1-11 possess either a cis or a trans fusion between rings $\mathrm{A}$ and $\mathrm{B}$, or a double bond between C-5 and C-6, leading to $5 \alpha$ (neotigogenin), $5 \beta$ (sarsasapogenin), and $\Delta^{5}$ (narthogenin) subtypes (Fig. 2). Neotigogenin glycosides $\mathbf{1 - 5}$, and $\mathbf{1 0}$ have been isolated from S. riparia [8], S. nipponica [9], and $S$. officinalis [7]. Both neotigogenin glycosides 5, 10 and sarsasapogenin glycoside $\mathbf{6}$ were identified from the rhizomes of S. officinalis [7]. Sarsasapogenin glycosides 7-9 were isolated from the root of $S$. aspera subsp. mauritanica [10], and S. ornata Lem. [11]. Compound 11, with a hydroxyl substitution on C-27, was the only narthogenin glycoside reported from Smilax species so far. galactopyranosyl moiety (Galp), a rhamnopyranosyl moiety (Rhap), a frutofuranosyl moiety (Furf) and an arabinopyranosyl moiety

\subsection{Isospirostane-Type Saponins $\mathbf{1 2 - 4 7}$}

Isospirostane-type saponins are also monodesmosidic glycosides characterized by an equatorial oriented methyl or hydroxymethyl (C-27) on F ring. The isospirostane-type saponins 12-47 could be classified into four subtypes on the basis of sapogenin structures, including diosgenin, laxogenin, tigogenin, and smilagenin (Fig. 3). The variations of these sapogenins mainly comprise dehydrogenation between $\mathrm{C}-5$ and $\mathrm{C}-6$, carbonylation at C-6, hydroxylation at $\mathrm{C}-17$ or $\mathrm{C}-27$, and cis/trans fusion between rings $\mathrm{A}$ and $\mathrm{B}$. Diosgenin glycosides 12-30 were characterized by a double bond between C- 5 and C- 6 . Diosgenin-3- $O-\alpha$-L-rhamnopyranoside (12) was the first diosgenin glycoside reported from the epigeal part of $S$. excelsa in 1975 [12]. Dioscin (13) was widely distributed among the Smilax species, including $S$. china [8], $S$. menispermoidea [13], S. lebrunii [14], S. nigrescens [15], S. stans [16], S. excels [17], S. microphylla [18], and $S$. bockii [19]. Parisyunnanosides C-E (18-20), with hydroxyl substitutions at C-7 or C-12, were isolated from the stems of $S$. riparia [20]. The occurrences of parisyunnanoside in the genus Smilax indicated the close chemtaxonomic relationship between the genus Smilax and Paris. Three isonarthogenin glycosides 24, 25, and 28 were isolated from $S$. scobinicaulis, together with two tigogenin 


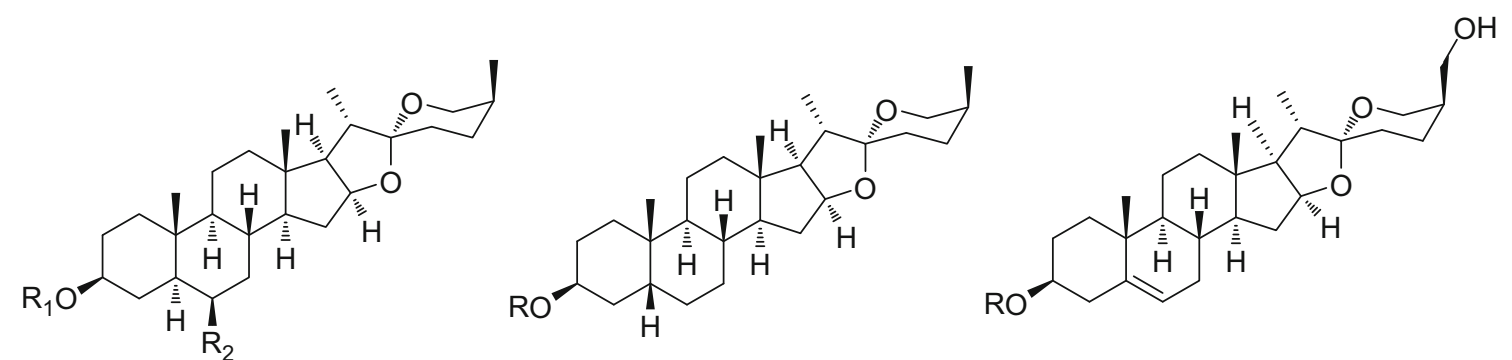

\begin{tabular}{lll}
\multicolumn{1}{l}{ neotigogenin } \\
\hline 1 & $R_{1}=S_{1}$ & $R_{2}=H$ \\
2 & $R_{1}=S_{2}$ & $R_{2}=H$ \\
3 & $R_{1}=S_{3}$ & $R_{2}=H$ \\
4 & $R_{1}=S_{4}$ & $R_{2}=H$ \\
5 & $R_{1}=S_{5}$ & $R_{2}=H$ \\
$10 R_{1}=S_{5}$ & $R_{2}=O H$
\end{tabular}
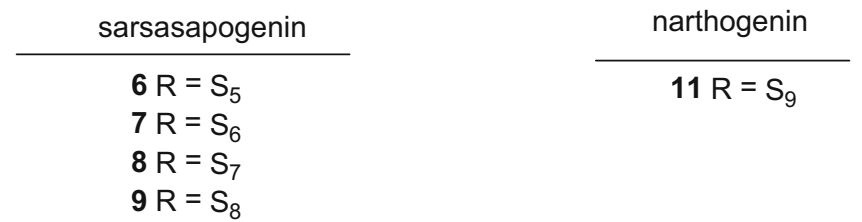

Fig. 2 Structures of compounds 1-11

glycosides 38-39 [21]. Sieboldogenin (33), with an additional hydroxyl substitution on C-27 in comparison with laxogenin, was identified from the ethyl acetate fraction of S. china [22]. Laxogenin glycosides $\mathbf{3 4 - 3 6}$ were founded in S. sieboldii [23]. Parisvietnaside A (37), characterized by a double bond between C-7 and C-8, was obtained from the roots and rhizomes of $S$. riparia [24]. The smilagenin glycosides 42-47 with a cis fusion rings $\mathrm{A}$ and $\mathrm{B}$ were isolated from the roots of $S$. medica [25, 26]. Hydroxyl substitution on C-7 or C-12, and double bond between C-7 and C-8 are the rare cases within the steroidal saponins of the genus Smilax.

\subsection{Furostane-Type Saponins $\mathbf{4 8 - 9 3}$}

Furostane-type saponins, F ring opened spirostanol glycosides, are another important group of steroidal saponins within Smilax species. The hemiketal hydroxy attached to the C-22 position of furostanol glycosides were sometimes methylated or dehydrated. The methylated derivatives were generally considered to be artifacts. Furostanol glycosides with both $25 R$ and $25 S$ configurations were reported from the genus Smilax. Additionally, furostanol glycosides always have two sugar chains attached to the C-3 and C-26 positions of the aglycone moiety (Fig. 4). Methylprotodioscin (48), protodioscin (59), and pseudoprotodioscin (60) were common constituents among the different Smilax species (Table 1). Compounds 50, isolated from the roots of $S$. bockii, increased the nerve growth factor (NGF)-induced neurite outgrowth in PC 12D cells by $49 \%$ in comparison with the blank control at the concentration of $60 \mu \mathrm{g} / \mathrm{mL}$ [19]. Compounds 53-55, identified from the rhizomes of $S$. excelsa, were the only three steroidal saponins with acylated sugars moieties within the genus
Smilax [17]. Furostane glycosides $\mathbf{6 2}$ and $\mathbf{6 3}$, with an oxygenated C-15, were isolated from the tubers of $S$. china [27]. Interestingly, the spirostane or isospirostane glycosides with an oxygenated C-15 have never been reported from Smilax so far. Compounds 67-70 with carbonylation on C-6 were isolated from the roots and rhizomes of $S$. scobinicaulis, together with a spirostane glycoside $\mathbf{3 5}$, and three furostane glycosides 89-91 [28]. Compounds 76 and 77, isolated from the root of $S$. officinalis, are the diastereoisomers with opposite configuration at C-5 [29]. Smilaxosides A-C $(\mathbf{8 4}, \mathbf{8 6}, \mathbf{8 7})$, and $(25 R)$-Smilaxchinoside A (85) were obtained tubers from $S$. china [30]. Of them, compounds 84 and $\mathbf{8 5}$ are diastereoisomers with opposite configuration at C-25. Compounds 92 and $\mathbf{9 3}$, identified from $S$. aspera [31], were rare examples with hydroxyl substitution on C-1 within the genus Smilax.

\subsection{Pregnane-Type Saponins 94-102 and Others 103- 104}

Pregane-type saponins are $\mathrm{C}_{21}$ steroidal saponins with a sugar moiety linked to the alcoholic hydroxyl group of the sapogenin, most frequently at C-3. Compounds 94-98 are not real pregnane-type saponins from the perspective of biosynthetic pathway. Possibly, they are biosynthetically formed through oxidative cleavage of the double bond between C-20 and C-22 in furostane structures. Compounds 94 and 98 were isolated from the rhizomes and roots of $S$. trinervula, together with compounds $11, \mathbf{6 0}, \mathbf{8 5}$, $\mathbf{8 8}$, and $\mathbf{1 0 3}$ [32]. Pregnane glycosides 99-102 were found in S. nigrescens [15], S. menispermoidea [33], S. bockii [19], S. microphylla [18], and S. riparia [20]. Compounds 103 and 104, isolated from $S$. trinervula and S. china respectively, are belonged to cholestane-type saponins, or 


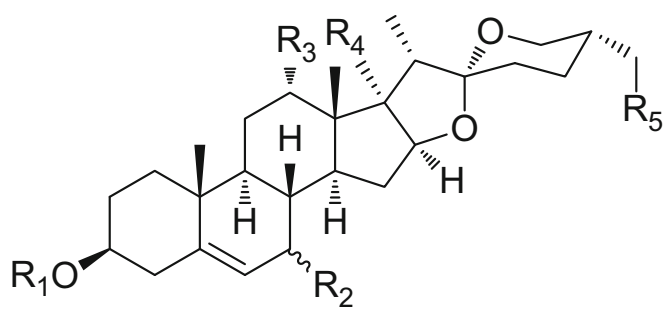

diosgenin

$12 \mathrm{R}_{1}=\mathrm{S}_{10} \mathrm{R}_{2}=\mathrm{R}_{3}=\mathrm{R}_{4}=\mathrm{R}_{5}=\mathrm{H}$

$13 \mathrm{R}_{1}=\mathrm{S}_{9} \quad \mathrm{R}_{2}=\mathrm{R}_{3}=\mathrm{R}_{4}=\mathrm{R}_{5}=\mathrm{H}$

$14 R_{1}=S_{11} R_{2}=R_{3}=R_{4}=R_{5}=H$

$15 R_{1}=S_{12} R_{2}=R_{3}=R_{4}=R_{5}=H$

$16 R_{1}=S_{13} R_{2}=R_{3}=R_{4}=R_{5}=H$

$17 \mathrm{R}_{1}=\mathrm{S}_{14} \mathrm{R}_{2}=\mathrm{R}_{3}=\mathrm{R}_{4}=\mathrm{R}_{5}=\mathrm{H}$

$18 \mathrm{R}_{1}=\mathrm{S}_{13} \mathrm{R}_{3}=\alpha_{-} \mathrm{OH} \mathrm{R}_{2}=\mathrm{R}_{4}=\mathrm{R}_{5}=\mathrm{H}$

$19 \mathrm{R}_{1}=\mathrm{S}_{15} \mathrm{R}_{2}=\alpha_{-} \mathrm{OH} \mathrm{R}_{3}=\mathrm{R}_{4}=\mathrm{R}_{5}=\mathrm{H}$

$20 \mathrm{R}_{1}=\mathrm{S}_{15} \mathrm{R}_{2}=\beta-\mathrm{OH} \mathrm{R}_{3}=\mathrm{R}_{4}=\mathrm{R}_{5}=\mathrm{H}$

$21 \mathrm{R}_{1}=\mathrm{S}_{14} \mathrm{R}_{4}=\mathrm{OH} \mathrm{R} \mathrm{R}_{2}=\mathrm{R}_{3}=\mathrm{R}_{5}=\mathrm{H}$

$22 \mathrm{R}_{1}=\mathrm{S}_{15} \mathrm{R}_{4}=\mathrm{OH} \mathrm{R}_{2}=\mathrm{R}_{3}=\mathrm{R}_{5}=\mathrm{H}$

$23 \mathrm{R}_{1}=\mathrm{S}_{9} \mathrm{R}_{2}=\mathrm{R}_{3}=\mathrm{HR}_{4}=\mathrm{R}_{5}=\mathrm{OH}$

$24 R_{1}=S_{16} R_{2}=R_{3}=H R_{4}=R_{5}=O H$

$25 \mathrm{R}_{1}=\mathrm{S}_{5} \quad \mathrm{R}_{2}=\mathrm{R}_{3}=\mathrm{HR}_{4}=\mathrm{R}_{5}=\mathrm{OH}$

$26 \mathrm{R}_{1}=\mathrm{S}_{11} \mathrm{R}_{2}=\mathrm{R}_{3}=\mathrm{HR}_{4}=\mathrm{R}_{5}=\mathrm{OH}$

$27 \mathrm{R}_{1}=\mathrm{S}_{22} \quad \mathrm{R}_{2}=\mathrm{R}_{3}=\mathrm{HR}_{4}=\mathrm{R}_{5}=\mathrm{OH}$

$28 R_{1}=S_{16} \quad R_{2}=R_{3}=R_{4}=H R_{5}=O H$

$29 \mathrm{R}_{1}=\mathrm{S}_{9} \quad \mathrm{R}_{2}=\mathrm{R}_{3}=\mathrm{R}_{4}=\mathrm{H} \mathrm{R}_{5}=\mathrm{OH}$

$30 \mathrm{R}_{1}=\mathrm{S}_{5} \quad \mathrm{R}_{2}=\mathrm{R}_{3}=\mathrm{R}_{4}=\mathrm{HR}_{5}=\mathrm{OH}$

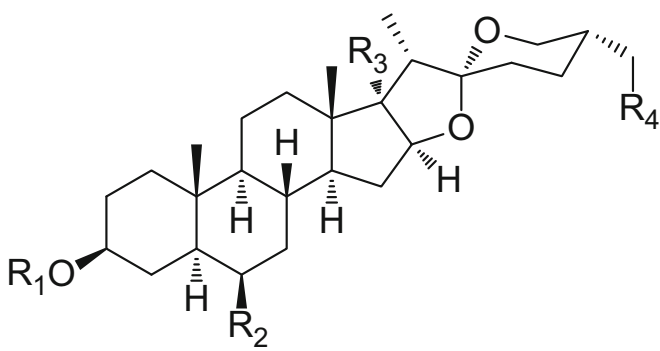

tigogenin

$38 \mathrm{R}_{1}=\mathrm{S}_{5} \mathrm{R}_{2}=\mathrm{R}_{3}=\mathrm{R}_{4}=\mathrm{H}$

$39 \mathrm{R}_{1}=\mathrm{S}_{5} \mathrm{R}_{2}=\mathrm{OH} \mathrm{R} \mathrm{R}_{3}=\mathrm{R}_{4}=\mathrm{H}$

$40 \mathrm{R}_{1}=\mathrm{S}_{5} \quad \mathrm{R}_{2}=\mathrm{R}_{3}=\mathrm{HR}_{4}=\mathrm{OH}$

$41 \mathrm{R}_{1}=\mathrm{S}_{5} \mathrm{R}_{2}=\mathrm{H} \mathrm{R} \mathrm{R}_{3}=\mathrm{R}_{4}=\mathrm{OH}$

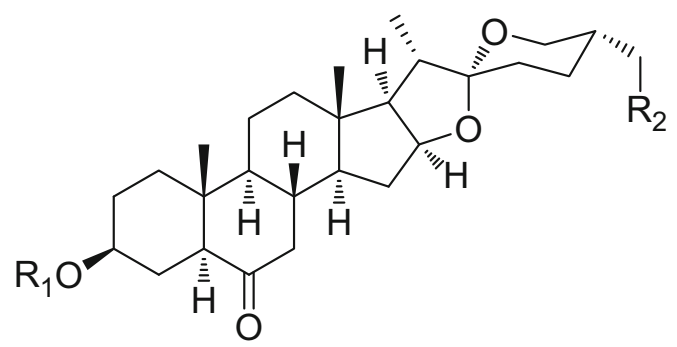

laxogenin

$31 \mathrm{R}_{1}=\mathrm{S}_{5} \quad \mathrm{R}_{2}=\mathrm{OH}$

$32 \mathrm{R}_{1}=\mathrm{S}_{16} \quad \mathrm{R}_{2}=\mathrm{OH}$

$33 \mathrm{R}_{1}=\mathrm{H} \quad \mathrm{R}_{2}=\mathrm{OH}$

$34 \mathrm{R}_{1}=\mathrm{S}_{17} \mathrm{R}_{2}=\mathrm{H}$

$35 \mathrm{R}_{1}=\mathrm{S}_{16} \mathrm{R}_{2}=\mathrm{H}$

$36 \mathrm{R}_{1}=\mathrm{S}_{5} \quad \mathrm{R}_{2}=\mathrm{H}$

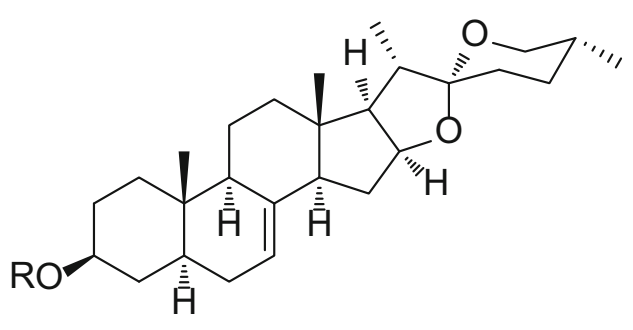

$37 \mathrm{R}=\mathrm{S}_{14}$

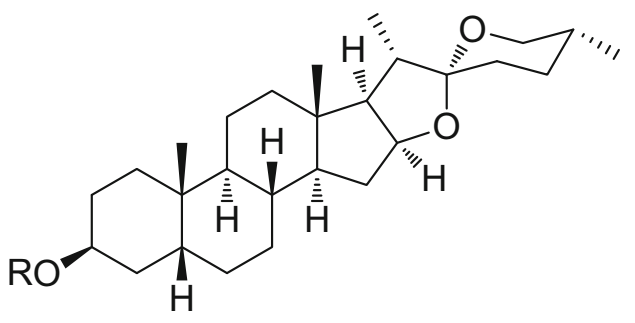

smilagenin

$42 \mathrm{R}=\mathrm{S}_{18}$

$43 \mathrm{R}=\mathrm{S}_{19}$

$44 \mathrm{R}=\mathrm{S}_{20}$

$45 \mathrm{R}=\mathrm{S}_{21}$

$46 \mathrm{R}=\mathrm{S}_{8}$

$47 \mathrm{R}=\mathrm{S}_{3}$

Fig. 3 Structures of compounds 12-47 


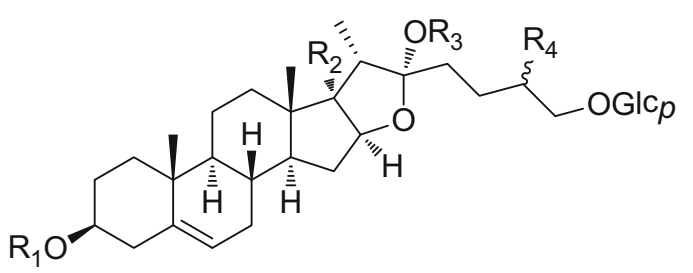

$48 \mathrm{R}_{1}=\mathrm{S}_{9} \mathrm{R}_{2}=\mathrm{HR}_{3}=\mathrm{CH}_{3} \quad \mathrm{R}_{4}=\beta-\mathrm{CH}_{3}$

$49 R_{1}=S_{12} R_{2}=H R_{3}=C_{3} R_{4}=\beta-C_{3}$

$50 \mathrm{R}_{1}=\mathrm{S}_{13} \mathrm{R}_{2}=\mathrm{HR}_{3}=\mathrm{CH}_{3} \mathrm{R}_{4}=\beta-\mathrm{CH}_{3}$

$51 R_{1}=S_{9} R_{2}=R_{3}=H R_{4}=\beta-C_{3}$

$52 R_{1}=S_{13} R_{2}=R_{3}=H R_{4}=\beta-C_{3}$

$53 R_{1}=S_{29} R_{2}=R_{3}=H R_{4}=\beta-C_{3}$

$54 R_{1}=S_{30} R_{2}=R_{3}=H R_{4}=\beta-C_{3}$

$55 R_{1}=S_{31} R_{2}=R_{3}=H R_{4}=\beta-C_{3}$

$56 \mathrm{R}_{1}=\mathrm{S}_{24} \mathrm{R}_{2}=\mathrm{OHR} \mathrm{R}_{3}=\mathrm{HR}_{4}=\beta-\mathrm{CH}_{3}$

$57 R_{1}=S_{14} R_{2}=R_{3}=H R_{4}=\alpha_{-} C H_{3}$

$58 \mathrm{R}_{1}=\mathrm{S}_{15} \mathrm{R}_{2}=\mathrm{R}_{3}=H \mathrm{R}_{4}=\alpha_{-} \mathrm{CH}_{3}$

$59 \mathrm{R}_{1}=\mathrm{S}_{15} \mathrm{R}_{2}=\mathrm{OH} \mathrm{R} \mathrm{R}_{3}=\mathrm{H} \mathrm{R} \mathrm{R}_{4}=\alpha-\mathrm{CH}_{3}$

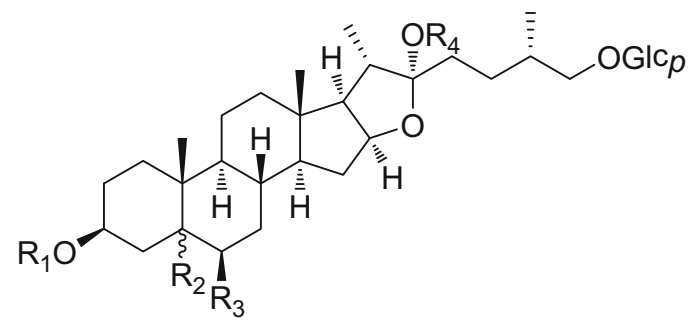

$71 \mathrm{R}_{1}=\mathrm{S}_{19} \mathrm{R}_{2}=\beta-\mathrm{H} \mathrm{R}_{3}=\mathrm{H} \mathrm{R} \mathrm{R}_{4}=\mathrm{CH}_{3}$

$72 \mathrm{R}_{1}=\mathrm{S}_{6} \quad \mathrm{R}_{2}=\beta-\mathrm{H} \quad \mathrm{R}_{3}=\mathrm{R}_{4}=\mathrm{H}$

$73 \quad R_{1}=S_{25} \quad R_{2}=\beta-H \quad R_{3}=R_{4}=H$

$74 R_{1}=S_{26} \quad R_{2}=\beta-H \quad R_{3}=H \quad R_{4}=C_{3}$

$75 \quad R_{1}=S_{26} \quad R_{2}=\beta-H R_{3}=R_{4}=H$

$76 \quad \mathrm{R}_{1}=\mathrm{S}_{5} \quad \mathrm{R}_{2}=\beta-\mathrm{H} \mathrm{R} \mathrm{R}_{3}=\mathrm{R}_{4}=\mathrm{H}$

$77 \quad R_{1}=S_{5} \quad R_{2}=\alpha_{-H} \quad R_{3}=R_{4}=H$

$78 \mathrm{R}_{1}=\mathrm{S}_{5} \quad \mathrm{R}_{2}=\alpha_{-} \mathrm{H} \quad \mathrm{R}_{3}=\mathrm{OH} \mathrm{R}_{4}=\mathrm{H}$

$79 \mathrm{R}_{1}=\mathrm{S}_{27} \quad \mathrm{R}_{2}=\beta-\mathrm{H} \quad \mathrm{R}_{3}=\mathrm{R}_{4}=\mathrm{H}$

$80 \quad R_{1}=S_{21} \quad R_{2}=\beta-H \quad R_{3}=R_{4}=H$

$81 R_{1}=S_{8} \quad R_{2}=\beta-H \quad R_{3}=R_{4}=H$

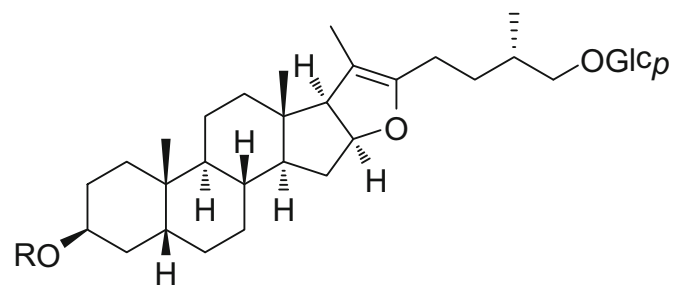

$82 \mathrm{R}=\mathrm{S}_{8}$

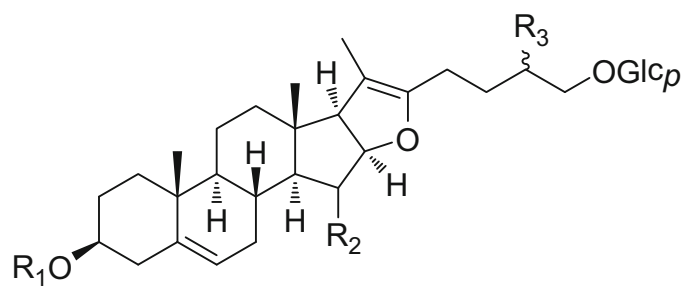

$60 \mathrm{R}_{1}=\mathrm{S}_{9} \mathrm{R}_{2}=\mathrm{H} \mathrm{R} \mathrm{R}_{3}=\beta-\mathrm{CH}_{3}$

$61 R_{1}=S_{12} R_{2}=H R_{3}=\beta-C_{3}$

$62 \mathrm{R}_{1}=\mathrm{S}_{9} \mathrm{R}_{2}=\mathrm{OH} \mathrm{R}_{3}=\beta-\mathrm{CH}_{3}$

$63 \mathrm{R}_{1}=\mathrm{S}_{9} \mathrm{R}_{2}=O \mathrm{OCH}_{3} \mathrm{R}_{3}=\beta-\mathrm{CH}_{3}$

$65 \mathrm{R}_{1}=\mathrm{S}_{23} \mathrm{R}_{2}=\mathrm{H} \mathrm{R} \mathrm{R}_{3}=\alpha-\mathrm{CH}_{3}$

$66 \mathrm{R}_{1}=\mathrm{S}_{15} \mathrm{R}_{2}=\mathrm{H} \mathrm{R} \mathrm{R}_{3}=\alpha_{-} \mathrm{CH}_{3}$

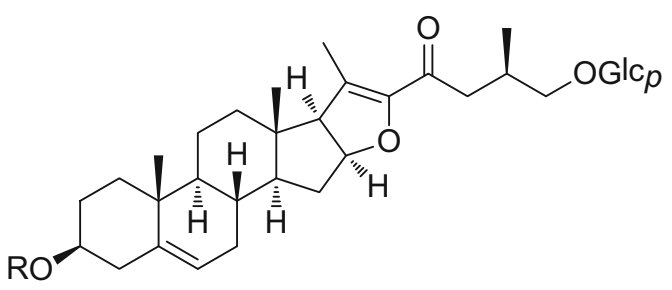

$64 \mathrm{R}=\mathrm{S}_{9}$

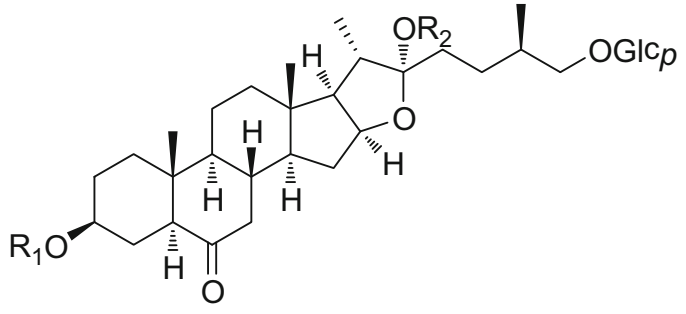

$67 \mathrm{R}_{1}=\mathrm{S}_{16} \quad \mathrm{R}_{2}=\mathrm{H}$

$68 \mathrm{R}_{1}=\mathrm{S}_{5} \mathrm{R}_{2}=\mathrm{H}$

$69 \mathrm{R}_{1}=\mathrm{S}_{16} \mathrm{R}_{2}=\mathrm{CH}_{3}$

$70 \mathrm{R}_{1}=\mathrm{H} \quad \mathrm{R}_{2}=\mathrm{CH}_{3}$<smiles>[R6][R5]=[As][R5]</smiles>

Fig. 4 Structures of compounds $48-83$ 
Table 1 Steroidal saponins from the Genus Smilax

\begin{tabular}{|c|c|c|c|c|}
\hline No. & Name & Plant & Parts & Ref. \\
\hline \multicolumn{5}{|c|}{ Spirostane-type saponin } \\
\hline \multirow[t]{2}{*}{1} & \multirow[t]{2}{*}{ Neotigogenin-3-O- $\alpha$-L-rhamnopyranosyl-( $\rightarrow$ 6)- $\beta$-D-glucopyranoside } & S. riparia & Rhizomes and roots & [8] \\
\hline & & S. lanceaefolia & Roots & [37] \\
\hline 2 & $\begin{array}{l}\text { Neotigogenin-3- } O \text { - } \beta \text {-D-glucopyranosyl- }(1 \rightarrow 4)-O \text { - }[\alpha \text {-L-rhamnopyranosyl- } \\
\quad(1 \rightarrow 6)]-\beta \text {-D-glucopyranoside }\end{array}$ & S. riparia & Rhizomes and roots & [8] \\
\hline 3 & Neotigogenin-3- $O$ - $\beta$-D-glucopyranoside & S. nipponica & Subterranean & [9] \\
\hline 4 & Smilanippin A & S. nipponica & Subterranean & [9] \\
\hline 5 & $\begin{array}{l}\text { Neotigogenin-3- } O \text { - } \beta \text {-D-glucopyranosyl- }(1 \rightarrow 4)-O \text { - }[\alpha \text {-L-arabinopyranosyl- } \\
\quad(1 \rightarrow 6)]-\beta \text {-D-glucopyranoside }\end{array}$ & S. officinalis & Rhizomes & [7] \\
\hline 6 & $\begin{array}{l}\text { Sarsasapogenin-3- } O \text { - } \beta \text {-D-glucopyranosyl- }(1 \rightarrow 4) \text {-[ } \alpha \text {-L-arabinopyranosyl- } \\
\quad(1 \rightarrow 6)]-\beta \text {-D-glucopyranoside }\end{array}$ & S. officinalis & Rhizomes & [7] \\
\hline 7 & $\begin{array}{l}(25 \mathrm{~S})-5 \beta \text {-Spirostane- } 3 \beta \text {-ol-3- } O \text { - } \alpha \text {-L-rhamnopyranosyl- }(1 \rightarrow 2)-\beta \text {-D- } \\
\text { glucopyranosyl- }(1 \rightarrow 2)-\beta \text {-D-glucopyranoside }\end{array}$ & S. aspera subsp. mauritanica & Roots & [10] \\
\hline 8 & Curillin G & S. aspera subsp. mauritanica & Roots & [10] \\
\hline 9 & Parillin & S. aristolochiifolia & Rhizomes and roots & [38] \\
\hline & & S. ornate & & [11] \\
\hline 10 & $\begin{array}{l}(25 S) \text {-Spirostan- } 6 \beta \text {-ol-3- } O-\beta \text {-D-glucopyranosyl- }(1 \rightarrow 4)-O \text { - }[\alpha-\mathrm{L}- \\
\quad \text { arabinopyranosyl- }(1 \rightarrow 6)]-\beta \text {-D-glucopyranoside }\end{array}$ & S. officinalis & Rhizomes & [7] \\
\hline 11 & Trinervuloside $\mathrm{C}$ & S. trinervula & Rhizomes and roots & [32] \\
\hline \multicolumn{5}{|c|}{ Isospirostane-type saponin } \\
\hline 12 & Diosgenin-3- $O-\alpha$-L-rhamnopyranoside & S. excels & Epigeal part & [12] \\
\hline \multirow[t]{9}{*}{13} & \multirow[t]{9}{*}{ Dioscin } & S. china & Roots & [8] \\
\hline & & S. menispermoides & Rhizomes & [13] \\
\hline & & S. lebrunii & Roots & [14] \\
\hline & & S. nigrescens & Roots & [15] \\
\hline & & S. stans & Roots & [16] \\
\hline & & S. bockii & Roots & [19] \\
\hline & & S. excelsa & Rhizomes & [17] \\
\hline & & S. microphylla & Tubers & [18] \\
\hline & & S. china & Tubers & [30] \\
\hline \multirow[t]{4}{*}{14} & \multirow[t]{4}{*}{ Diosgenin-3- $O$ - $[\alpha$-L-rhamnopyranosyl- $(1 \rightarrow 4)]-\beta$-D-glucopyranoside } & S. nigrescens & Roots & [15] \\
\hline & & S. menispermoides & Roots & [39] \\
\hline & & S. menispermoides & Rhizomes & [33] \\
\hline & & S. china & Tubers & [30] \\
\hline \multirow[t]{3}{*}{15} & \multirow[t]{3}{*}{ Diosgenin-3- $O$ - $[\alpha$-L-rhamnopyranosyl- $(1 \rightarrow 2)]-\beta$-D-glucopyranoside } & S. nigrescens & Roots & [15] \\
\hline & & S. menispermoides & Rhizomes & [33] \\
\hline & & S. microphylla & Tubers & [18] \\
\hline 16 & $\begin{array}{l}(25 R) \text {-Spirostan-5-en-3- } O-\alpha \text {-L-rhamnopyranosyl- }(1 \rightarrow 2)-[\alpha \text {-L- } \\
\text { rhamnopyranosyl- }(1 \rightarrow 4)-\alpha \text {-L-rhamnopyranosyl- }(1 \rightarrow 4)]-O-\beta \text {-D- } \\
\text { glucopyranoside }\end{array}$ & S. china & Tubers & {$[30]$} \\
\hline 17 & Gracillin & S. microphylla & Tubers & [18] \\
\hline 18 & Parisyunnanoside $\mathrm{C}$ & S. riparia & Rhizomes and roots & {$[20]$} \\
\hline 19 & Parisyunnanoside D & S. riparia & Rhizomes and roots & {$[20]$} \\
\hline 20 & Parisyunnanoside E & S. riparia & Rhizomes and roots & {$[20]$} \\
\hline 21 & Paris D & S. riparia & Rhizomes and roots & {$[20]$} \\
\hline 22 & Paris H & S. riparia & Rhizomes and roots & {$[20]$} \\
\hline 23 & $\begin{array}{l}(25 R) \text {-Spirost-5-en-3 } \beta, 17 \alpha, 27 \text {-triol-3- } O \text { - } \alpha \text {-L-rhamnopyranosyl-(l } \rightarrow 2) \text {-[ }[\alpha \text {-L- } \\
\text { rhamnopyranosyl- }(1 \rightarrow 4)] \text { - } \beta \text {-D-glucopyranoside }\end{array}$ & S. menispermoides & Rhizomes & {$[40]$} \\
\hline
\end{tabular}


Table 1 continued

\begin{tabular}{|c|c|c|c|c|}
\hline No. & Name & Plant & Parts & Ref. \\
\hline \multirow[t]{3}{*}{24} & \multirow{3}{*}{$\begin{array}{l}\text { (25S)-Spirostan-5-en-3 } \beta, 17 \alpha, 27 \text {-triol-3- } O-\alpha \text {-L-arabinopyranosyl- }(1 \rightarrow 6)-\beta \text { - } \\
\text { D-glucopyranoside }\end{array}$} & S. lebrunii & Roots & [14] \\
\hline & & S. lebrunii & Roots & [41] \\
\hline & & S. scobinicaulis & Rhizomes and roots & [21] \\
\hline \multirow[t]{4}{*}{25} & \multirow{4}{*}{$\begin{array}{l}(25 S) \text {-Spirostan-5-en-3 } \beta, 17 \alpha, 27 \text {-triol-3- } O \text { - } \beta \text {-D-glucopyranosyl- }(1 \rightarrow 4) \text { - }[\alpha \text {-L- } \\
\quad \text { arabinopyranosyl- }(1 \rightarrow 6)]-\beta \text {-D-glucopyranoside }\end{array}$} & S. lebrunii & Rhizomes & [33] \\
\hline & & S. lebrunii & Rhizomes & [42] \\
\hline & & S. scobinicaulis & Rhizomes and roots & [21] \\
\hline & & S. scobinicaulis & Rhizomes & [43] \\
\hline \multirow[t]{2}{*}{26} & \multirow{2}{*}{$\begin{array}{l}\text { (25S)-spirost-5-ene-3 } \beta, 17 \alpha, 27 \text {-triol-3- } O \text { - } \alpha \text {-L-rhamnopyranosyl-(1 } \rightarrow 4)-\beta \text {-D- } \\
\text { glucopyranoside }\end{array}$} & S. menispermoides & Roots & [39] \\
\hline & & S. menispermoides & Rhizomes & [33] \\
\hline 27 & (25S)-Spirost-5-en-3 $\beta, 17 \alpha, 27$-triol-3- $O-\beta$-D-galactopyranoside & S. menispermoides & Rhizomes & [33] \\
\hline \multirow[t]{2}{*}{28} & \multirow{2}{*}{$\begin{array}{l}\text { (25S)-Spirostan-5-en-3 } \beta, 27 \text {-diol-3- } O-\alpha \text {-L-arabinopyranosyl- }(1 \rightarrow 6)-\beta \text {-D- } \\
\text { glucopyranoside }\end{array}$} & S. scobinicaulis & Rhizomes and roots & [21] \\
\hline & & S. lebrunii & Roots & [14] \\
\hline \multirow[t]{2}{*}{29} & \multirow{2}{*}{$\begin{array}{l}\text { Isonarthogenin3- } O \text { - } \alpha \text {-L-rhamnopyranosyl- }(1 \rightarrow 2) \text { - } O \text {-[ } \alpha \text {-L-rhamnopyranosyl- } \\
\quad(1 \rightarrow 4)] \text { - } \beta \text {-D-glucopyranoside }\end{array}$} & S. china & Roots & [8] \\
\hline & & S. china & Tubers & [30] \\
\hline 30 & Smilscobinoside A & S. scobinicaulis & Rhizomes and roots & [44] \\
\hline \multirow[t]{3}{*}{31} & \multirow[t]{3}{*}{ Sieboldiin A } & S. sieboldii & Subterranean & [45] \\
\hline & & S. sieboldii & Rhizomes & [23] \\
\hline & & S. scobinicaulis & Rhizomes & [46] \\
\hline \multirow[t]{4}{*}{32} & \multirow[t]{4}{*}{ Sieboldiin B } & S. sieboldii & Subterranean & [45] \\
\hline & & S. sieboldii & Rhizomes & [23] \\
\hline & & S. scobinicaulis & Rhizomes & [46] \\
\hline & & S. scobinicaulis & Rhizomes and roots & [28] \\
\hline 33 & Sieboldogenin & S. china & Rhizomes & [22] \\
\hline \multirow[t]{2}{*}{34} & \multirow{2}{*}{$\begin{array}{l}(25 R)-5 \alpha \text {-Spirostan-3 } \beta \text {-ol-6-one-3- } O \text { - }[\alpha \text {-L-arabinopyranosyl- }(1 \rightarrow 4)]-\beta \text {-D- } \\
\quad \text { glucopyranoside }\end{array}$} & S. lebrunii & Roots & [14] \\
\hline & & S. lebrunii & Roots & [41] \\
\hline \multirow[t]{3}{*}{35} & \multirow[t]{3}{*}{ Smilaxin A } & S. sieboldii & Subterranean & [45] \\
\hline & & S. lebrunii & Rhizomes & [47] \\
\hline & & S. scobincaulis & Rhizomes & [48] \\
\hline \multirow[t]{4}{*}{36} & \multirow[t]{4}{*}{ Smilaxin B } & S. sieboldii & Subterranean & [45] \\
\hline & & S. lebrunii & Rhizomes & [47] \\
\hline & & S. sieboldii & Rhizomes & [23] \\
\hline & & S. scobincaulis & Rhizomes & [48] \\
\hline 37 & Parisvietnaside A & S. riparia & Rhizomes and roots & [20] \\
\hline \multirow[t]{3}{*}{38} & \multirow[t]{3}{*}{ Smilaxin $\mathrm{C}$} & S. sieboldii & Subterranean & [45] \\
\hline & & S. sieboldii & Rhizomes & [23] \\
\hline & & S. scobinicaulis & Rhizomes and roots & [21] \\
\hline 39 & $\begin{array}{l}(25 R)-5 \alpha \text {-Spirostan-3 } \beta, 6 \beta \text {-diol-3- } O-\beta \text {-D-glucopyranosyl- }(1 \rightarrow 4)-[\alpha-\mathrm{L}- \\
\quad \text { arabinopyranosyl- }(1 \rightarrow 6)]-\beta \text {-D-glucopyranoside }\end{array}$ & S. scobinicaulis & Rhizomes and roots & [21] \\
\hline 40 & Smilscobinoside B & S. scobinicaulis & Rhizomes and roots & [44] \\
\hline \multirow[t]{2}{*}{41} & \multirow{2}{*}{$\begin{array}{l}(25 R)-5 \alpha \text {-Spirostan-3 } \beta, 17 \alpha, 27 \text {-triol-3- } O \text { - } \beta \text {-D-glucopyranosyl- }(1 \rightarrow 4)-[\alpha-\mathrm{L}- \\
\quad \text { arabinopyranosyl- }(1 \rightarrow 6)]-\beta \text {-D-glucopyranosie }\end{array}$} & S. scobinicaulis & Rhizomes & [43] \\
\hline & & S. scobinicaulis & Rhizomes and roots & [44] \\
\hline 42 & $\begin{array}{l}(25 R)-5 \beta \text { Spirostan-3 } \beta \text {-ol-3- } O \text { - } \beta \text {-D-glucopyranosyl- }(1 \rightarrow 6) \text {-[ } \beta \text {-D- } \\
\text { glucopyranosyl- }(1 \rightarrow 4)]-\beta \text {-D-glucopyranoside }\end{array}$ & S. medica & Rhizomes & {$[25]$} \\
\hline 43 & $\begin{array}{l}(25 R)-5 \beta \text {-Spirostan-3 } \beta \text {-ol-3- } O \text { - } \beta \text {-D-glucopyranosyl- }(1 \rightarrow 6)-[\beta \text {-D- } \\
\text { glucopyranosyl- }(1 \rightarrow 2)]-[\beta \text {-D-glucopyranosyl- }(1 \rightarrow 4)]-\beta \text {-D- } \\
\text { glucopyranoside }\end{array}$ & S. medica & Rhizomes & {$[25]$} \\
\hline 44 & Disporoside A & S. medica & Rhizomes & {$[25]$} \\
\hline 45 & $\begin{array}{l}(25 R)-5 \beta \text {-Spirostan-3 } \beta \text {-ol-3- } O \text { - } \beta \text {-D-glucopyranosyl- }(1 \rightarrow 6)-\beta \text {-D- } \\
\text { glucopyranoside }\end{array}$ & S. medica & Rhizomes & [26] \\
\hline
\end{tabular}


Table 1 continued

\begin{tabular}{|c|c|c|c|c|}
\hline No. & Name & Plant & Parts & Ref. \\
\hline 46 & $\begin{array}{l}(25 R)-5 \beta \text {-Spirostan-3 } \beta \text {-ol-3- } O \text { - } \beta \text {-D-glucopyranosyl- }(1 \rightarrow 6) \text { - }[\beta \text {-D- } \\
\text { glucopyranosyl- }(1 \rightarrow 2)] \text { - }[\alpha \text {-L-rhamnopyranosyl- }(1 \rightarrow 4)]-\beta \text {-D- } \\
\text { glucopyranoside }\end{array}$ & S. medica & Rhizomes & {$[26]$} \\
\hline 47 & Smilagenin $3-O-\beta$-D-glucopyranoside & S. medica & Rhizomes & [26] \\
\hline \multicolumn{5}{|c|}{ Furostane-type saponin } \\
\hline \multirow[t]{7}{*}{48} & \multirow[t]{7}{*}{ Methylprotodioscin } & S. china & Roots & {$[8]$} \\
\hline & & S. menispermoides & Rhizomes & [13] \\
\hline & & S. stans & Roots & {$[16]$} \\
\hline & & S. bockii & Roots & [19] \\
\hline & & S. microphylla & Tubers & {$[18]$} \\
\hline & & S. china & Tubers & {$[30]$} \\
\hline & & S. nigrescens & Roots & [49] \\
\hline 49 & $\begin{array}{l}\text { 26- } O \text { - } \beta \text {-D-Glucopyranosyl-(25R)-furostan-5-en-3 } \beta \text {,26-diol-22-methoxy-3- } O \text { - } \\
\alpha \text {-L-rhamnopyranosyl-( } \rightarrow \text { 2)- } \beta \text {-D-glucopyranoside }\end{array}$ & S. nigrescens & Roots & [49] \\
\hline 50 & $\begin{array}{l}\text { 26- } O \text { - } \beta \text {-D-Glucopyranosyl-22 } \alpha \text { - } O \text {-methyl-(25R)-furost-5-en-3 } \beta, 26 \text {-diol-3- } O \text { - } \\
\alpha \text {-L-rhamnopyranosyl- }(1 \rightarrow 4) \text { - } \alpha \text {-L-rhamnopyranosyl- }(1 \rightarrow 4) \text { - }[\alpha \text {-L- } \\
\text { rhamnopyranosyl- }(1 \rightarrow 2)]-\beta \text {-D-glucopyranoside }\end{array}$ & S. bockii & Roots & [19] \\
\hline \multirow[t]{3}{*}{51} & \multirow[t]{3}{*}{ Protodioscin } & S. excelsa & Rhizomes & [17] \\
\hline & & S. microphylla & Tubers & {$[18]$} \\
\hline & & S. china & Tubers & {$[30]$} \\
\hline 52 & $\begin{array}{l}\text { Protodiosgenin-3- } O \text { - } \alpha \text {-L-rhamnopyranosyl- }(1 \rightarrow 4)-\alpha \text {-L- } \\
\text { rhamnopyranosyl }(1 \rightarrow 4) \text {-[ } \alpha \text {-L-rhamnopyranosyl }(1 \rightarrow 2)]-\beta \text {-D- } \\
\text { glucopyranoside }\end{array}$ & S. krausiana & Rhizomes & {$[50]$} \\
\hline 53 & $\begin{array}{l}\text { 26- } O \text { - } \beta \text {-D-Glucopyranosyl-22 } \alpha \text {-hydroxy- }(25 R) \text {-furost- } 5 \text {-en-3 } \beta, 26 \text {-diol-3- } O \text { - } \\
\text { [4- } O \text {-acetyl- } \alpha \text {-L-rhamnopyranosyl]- }(1 \rightarrow 2) \text { - }[\alpha \text {-L-rhamnopyranosyl- } \\
\quad(1 \rightarrow 4)]-\beta \text {-D-glucopyranoside }\end{array}$ & S. excelsa & Rhizomes & [17] \\
\hline 54 & $\begin{array}{l}\text { 26- } O \text { - } \beta \text {-D-Glucopyranosyl-22 } \alpha \text {-hydroxy-(25R)-furost-5-en-3 } \beta, 26 \text {-diol-3- } O \text { - } \\
\text { [2- } O \text {-acetyl- } \alpha \text {-L-rhamnopyranosyl]-( } \rightarrow 2) \text {-[ } \alpha \text {-L-rhamnopyranosyl- } \\
\quad(1 \rightarrow 4)]-\beta \text {-D-glucopyranoside }\end{array}$ & S. excelsa & Rhizomes & [17] \\
\hline 55 & $\begin{array}{l}\text { 26- } O \text { - } \beta \text {-D-Glucopyranosyl-22 } \alpha \text {-hydroxy- }(25 R) \text {-furost-5-en-3 } \beta, 26 \text {-diol-3- } O \text { - } \\
\text { [3- } O \text {-acetyl- } \alpha \text {-L-rhamnopyranosyl]- }(1 \rightarrow 2) \text { - }[\alpha \text {-L-rhamnopyranosyl- } \\
\quad(1 \rightarrow 4)]-\beta \text {-D-glucopyranoside }\end{array}$ & S. excelsa & Rhizomes & [17] \\
\hline 56 & $\begin{array}{l}\text { 26- } O \text { - } \beta \text {-D-Glucopyranosyl-( } 25 R) \text {-furostan-5-en-3 } \beta, 17 \alpha \text {-diol-3- } O-\alpha \text {-L- } \\
\text { rhamnopyranosyl-( }(\rightarrow 2)-\alpha \text {-L-rhamnopyranoside }\end{array}$ & S. scobiniculis & Rhizomes & {$[51]$} \\
\hline 57 & Protogracillin & S. riparia & Rhizomes and roots & {$[20]$} \\
\hline 58 & Parisaponin I & S. riparia & Rhizomes and roots & {$[20]$} \\
\hline 59 & Parisyunnanoside A & S. riparia & Rhizomes and roots & {$[20]$} \\
\hline \multirow[t]{7}{*}{60} & \multirow[t]{7}{*}{ Pseudoprotodioscin } & S. china & Roots & [8] \\
\hline & & S. trinervula & Rhizomes and roots & {$[32]$} \\
\hline & & S. menispermoides & Rhizomes & {$[13]$} \\
\hline & & S. stans & Roots & {$[16]$} \\
\hline & & S. excelsa & Rhizomes & {$[17]$} \\
\hline & & S. china & Tubers & {$[30]$} \\
\hline & & S. nigrescens & Roots & [49] \\
\hline 61 & $\begin{array}{l}\text { 26- } O \text { - } \beta \text {-D-Glucopyranosyl-( } 25 R) \text {-furostan-5,20(22)-dien-3 } \beta, 26 \text {-diol-3- } O \text { - } \alpha \text {-L- } \\
\text { rhamnopyranosyl- }(1 \rightarrow 2) \text { - } \beta \text {-D-glucopyranoside }\end{array}$ & S. nigrescens & Roots & [49] \\
\hline 62 & 15-Hydroxypseudoprotodioscin & S. china & Tubers & {$[27]$} \\
\hline 63 & 15-Methoxypseudoprotodioscin & S. china & Tubers & {$[27]$} \\
\hline 64 & 23-Oxopseudoprotodioscin & S. microphylla & Tubers & {$[18]$} \\
\hline 65 & $\begin{array}{l}\text { 26- } O \text { - } \beta \text {-D-Glucopyranosyl-(25S)-5-furosa-20(22)-en-3 } \beta, 26 \text {-diol-3- } O-\alpha \text {-L- } \\
\text { rhamnopyranosyl- }(1 \rightarrow 2)-O \text { - }[\alpha \text {-L-rhamnopyranosyl-( } \rightarrow \text { - })]-\beta \text {-D- } \\
\text { glucopyranoside }\end{array}$ & S. riparia & Roots & {$[52]$} \\
\hline
\end{tabular}


Table 1 continued

\begin{tabular}{|c|c|c|c|c|}
\hline No. & Name & Plant & Parts & Ref. \\
\hline 66 & Pseudoproto-pb & S. riparia & Rhizomes and roots & [20] \\
\hline 67 & $\begin{array}{l}\text { 26- } O \text { - } \beta \text {-D-Glucopyranosyl-(25R)-5 } \alpha \text {-furostan-3 } \beta, 22,26 \text {-triol-6-one-3- } O-\alpha \text {-L- } \\
\text { arabinopyranosyl- }(1 \rightarrow 6)-\beta \text {-D-glucopyranoside }\end{array}$ & $\begin{array}{l}\text { S. sieboldii } \\
\text { S. scobinicaulis }\end{array}$ & $\begin{array}{l}\text { Rhizomes } \\
\text { Rhizomes and roots }\end{array}$ & $\begin{array}{l}{[23]} \\
{[28]}\end{array}$ \\
\hline 68 & $26-O$ - $\beta$-D-Glucopyranosyl-(25R)- $5 \alpha$-furostan- $3 \beta, 22,26$-triol-6-one-3- $O-\beta$-D- & S. sieboldii & Rhizomes & [23] \\
\hline
\end{tabular}

$6926-O-\beta$-D-Glucopyranosyl-(25R)-5 $\alpha$-furostan-3 $\beta$,26-diol-22-methoxyl-6-oneglucopyranosyl-( $1 \rightarrow 4)$-[ $\alpha$-L-arabinopyranosyl- $(1 \rightarrow 6)]-\beta$-Dglucopyranoside 3- $O$ - $\alpha$-L-arabinopyranosyl- $(1 \rightarrow 6)$ - $\beta$-D-glucopyranoside

$70 \quad 26-O-\beta$-D-Glucopyranosyl-(25R)-5 $\alpha$-furostan-3 $\beta, 26$-diol-22-methoxyl-6-one

S. scobinicaulis

Rhizomes and roots

$7126-O-\beta$-D-Glucopyranosyl-(25S)-5 $\beta$-furostan-3 $\beta, 26$-diol-22 $\alpha$-methoxy-3- $O$ $\beta$-D-glucopyranosyl-( $1 \rightarrow 6)$-[ $\beta$-D-glucopyranosyl- $(1 \rightarrow 2)]$-[ $\beta$-Dglucopyranosyl- $(1 \rightarrow 4)]$ - $\beta$-D-glucopyranoside

72 (25S)-26- $O$ - $\beta$-D-glucopyranosyl-3 $\beta, 5 \beta, 22 \alpha$-furostan-3,22,26-triol-3- $O-\alpha$-Lrhamnopyranosyl-( $\rightarrow 2$ )- $O$ - $\beta$-D-glucopyranosyl-( $\rightarrow 2)-O-\beta$-Dglucopyranoside

73 Asparagoside E

S. scobinicaulis

Rhizomes and roots

S. medica

S. aspera subsp. mauritanica Roots

S. aspera subsp. mauritanica Roots

S. aspera subsp. mauritanica Roots

74 Asparoside A

75 Asparoside B

$7626-O-\beta$-D-Glucopyranosyl-(25S)-5 $\beta$-furostan- $3 \beta, 22 \alpha$-diol-3- $O$ - $\alpha$-Larabinopyranosyl-(1 $\rightarrow 6)$-[ $\beta$-D-glucopyranosyl- $(1 \rightarrow 4)]-\beta$-Dglucopyranoside

$7726-O-\beta$-D-Glucopyranosyl-(25S)-5 $\alpha$-furostan-3 $\beta, 22 \alpha-$ diol-3- $O-\alpha$-Larabinopyranosyl-(1 $\rightarrow 6)$-[ $\beta$-D-glucopyranosyl- $(1 \rightarrow 4)]-\beta$-Dglucopyranoside

$7826-O-\beta$-D-Glucopyranosyl-(25S)-5 $\alpha$-furostan-3 $\beta, 6 \beta, 22 \alpha$-tetraol-3- $O$ - $\alpha$-L-

S. aspera subsp. mauritanica Roots

S. officinalis

Roots arabinopyranosyl-(1 $\rightarrow 6)$-[ $\beta$-D-glucopyranosyl-( $1 \rightarrow 4)]$ - $\beta$-D-glucopyrano side

79 Sarsaparilloside B

S. officinalis

Roots

S. officinalis

Roots

80 Sarsaparilloside C

S. ornate

Rhizomes and roots

81 Sarsaparilloside

S. ornate

Rhizomes and roots

$82 \quad \Delta^{20(22)}$-Sarsaparilloside

S. ornate

Rhizomes and roots

S. ornate

Rhizomes and roots

83 Riparoside A

S. riparia

Rhizomes and roots

84 Smilaxchinoside A

S. china

Tubers

S. china

Tubers

S. riparia

Rhizomes and roots

S. riparia

S. trinervula

Roots

[32]

S. china

Rhizomes and roots

86 Smilaxchinoside B

S. china

Tubers

S. riparia

Tubers

S. trinervula

Rhizomes and roots

88 Dioscoreside E

S. scobinicaulis

Rhizomes and roots

$90 \quad(23 R, 25 R)-5 \alpha$-Furostan-3 $\beta, 23,26$-triol-20(22)-en-6-one-26- $O$ - $\beta$-D-

S. scobinicaulis

Rhizomes and roots glucopyranoside

$91 \quad 26-O-\beta$-D-Glucopyranosyl-(25R)-5 $\alpha$-furostan-3 $\beta, 26$-diol-20(22)-en-6-one-3$O$ - $\alpha$-L-arabinopyranosyl- $(1 \rightarrow 6)-\beta$-D-glucopyranoside

92 (25S)-5 $\beta$-Furostan- $1 \beta, 2 \beta, 3 \beta, 5 \beta, 22 \alpha, 26$-hexaol-26- $O$ - $\beta$-D-glucopyrano side

S. scobinicaulis

Rhizomes and roots

Rhizomes and roots

$9326-O$ - $\beta$-D-Glucopyranosyl-(25S)-5 $\beta$-furostan- $1 \beta, 3 \beta, 22 \alpha, 26$-tetraol-1- $O$ - $\beta$-Dglucopyranoside

Rhizomes

Pregane-type saponin

94 Trinervuloside A

S. trinervula

Rhizomes and roots 
Table 1 continued

\begin{tabular}{|c|c|c|c|c|}
\hline No. & Name & Plant & Parts & Ref. \\
\hline \multirow[t]{3}{*}{95} & \multirow[t]{3}{*}{ Riparoside B } & S. riparia & Rhizomes and roots & {$[20]$} \\
\hline & & S. riparia & Rhizomes and roots & {$[55]$} \\
\hline & & S. riparia & Rhizomes and roots & {$[53]$} \\
\hline \multirow[t]{2}{*}{96} & \multirow[t]{2}{*}{ Timosaponin $\mathrm{J}$} & S.riparia & Rhizomes and roots & {$[20]$} \\
\hline & & S. riparia & Rhizomes and roots & {$[55]$} \\
\hline 97 & Timosaponin $\mathrm{K}$ & S. riparia & Rhizomes and roots & {$[20]$} \\
\hline 98 & Trinervuloside B & S. trinervula & Rhizomes and roots & {$[32]$} \\
\hline \multirow[t]{3}{*}{99} & \multirow{3}{*}{$\begin{array}{l}\text { Pregna-5,16-diene-3 } \beta \text {-ol-20-one-3- } O \text { - } \alpha \text {-L-rhamnopyranosyl-( }(\rightarrow 2)-O \text {-[ } \alpha \text {-L- } \\
\quad \text { rhamnopyranosyl- }(1 \rightarrow 4)]-\beta \text {-D-glucopyranoside }\end{array}$} & S. nigrescens & Roots & [15] \\
\hline & & S. bockii & Roots & [19] \\
\hline & & S. menispermoides & Rhizomes & {$[33]$} \\
\hline 100 & $\begin{array}{l}\text { Pregna-5,16-diene-3 } \beta \text {-ol-20-one-3- } O-\alpha \text {-L-rhamnopyranosyl- }(1 \rightarrow 4)-\alpha \text {-L- } \\
\text { rhamnopyranosyl- }(1 \rightarrow 4) \text {-[ } \alpha \text {-L-rhamnopyranosyl- }(1 \rightarrow 2)]-\beta \text {-D- } \\
\text { glucopyranoside }\end{array}$ & S. bockii & Roots & [19] \\
\hline 101 & $\begin{array}{l}\text { Pregna-5,16-diene- } 3 \beta \text {-ol-20-one-3- } O-\alpha \text {-L-rhamnopyranosyl- }(1 \rightarrow 2) \text {-[ } \alpha \text {-L- } \\
\text { rhamnopyranosyl- }(1 \rightarrow 4)]-\alpha \text {-L-rhamnopyranosyl- }(1 \rightarrow 2)-\beta \text {-D- } \\
\text { glucopyranoside }\end{array}$ & S. microphylla & Tubers & {$[18]$} \\
\hline 102 & Pallidfloside D & S. riparia & Rhizomes and roots & {$[20]$} \\
\hline \multicolumn{5}{|c|}{ Cholestane-type saponin } \\
\hline 103 & Anguivioside XV & S. trinervula & Rhizomes and roots & {$[32]$} \\
\hline 104 & Smilaxchinoside D & S. china & Tubers & {$[30]$} \\
\hline
\end{tabular}

open chain saponins in another way of saying [34]. $S$. riparia saponins, from which compounds 18-22, 57-59, 66, 85, 87, 95-97, and 102 were identified, exhibited the synergistic effects with allopurinolin in reducing serum uric acid levels and increasing the urine uric acid level in a hyperuricemic mouse model [20]. The attenuation of hyperuricemia-induced renal dysfunction was linked to the inhibition of serum and hepatic xanthine oxidase, the down-regulation of renal mURAT1 and GLUT9, and the up-regulation of mOAT1. Structures of steroidal saponins (94-104) are shown in Fig. 5.

\section{Biological Activities of Steroidal Saponins}

Steroidal saponins are considered to be responsible for pharmacological properties of Smilax species. Many pharmacological in vitro and in vivo studies revealed significant biological activities, including cAMP phosphodiesterase inhibitory, anti-fungal, cytotoxic, and antiinflammatory activities.

\section{1 cAMP Phosphodiesterase Inhibitory Activity}

The cAMP phosphodiesterase is an enzyme that degrades the phosphodiester bond in the second messenger molecule cAMP. It regulates the localization, duration, and amplitude of cyclic nucleotide signaling within subcellular domains. Compounds 1, 2, 29 and 60, showed cAMP phosphodiesterase inhibitory activities with $\mathrm{IC}_{50}$ values of $102,55,93$, and $47 \mu \mathrm{M}$, respectively, which were almost equal to that of positive control papaverine $\left(\mathrm{IC}_{50}=30 \mu \mathrm{M}\right)$ [8]. Laxogenin glycosides 34, 35, and isospirostanol glycoside 38 displayed cAMP phosphodiesterase inhibitory activities with $\mathrm{IC}_{50}$ values of 83,34 , and $32 \mu \mathrm{M}$, respectively. While compound 36, with an additional hydroxyl substitution on C-27 in comparison with compound 34, showed no obvious inhibitory activity. Furostane glycosides 67-68 were inactive [23].

\subsection{Antifungal Activity}

$\mathrm{C}_{27}$ steroidal glycosides are well known for their antifungal activities [35]. Sarsasapogenin glycosides 7, 8, and four furostane glycosides 72-75, were tested for their antifungal activity. Compound $\mathbf{8}$ showed antifungal activity against three human pathogenic species, Candida albicans, $C$. glabrata, and $C$. tropicalis, with minimal inhibitory concentration (MIC) values of 25,25 and $50 \mu \mathrm{g} / \mathrm{mL}$, respectively. While compounds $\mathbf{7}$ and 72-75 showed no obvious antifungal activity at concentration of $200 \mu \mathrm{g} / \mathrm{mL}$ [10]. Six smilagenin glycosides $\mathbf{4 2 - 4 7}$ and a furostane glycoside $\mathbf{7 1}$ were also evaluated for their antifungal activities against these three pathogenic species. Compounds 42-46 demonstrated moderate antifungal activity with MIC values between 12.5 and $50 \mu \mathrm{g} / \mathrm{mL}[25,26]$. With regard to 


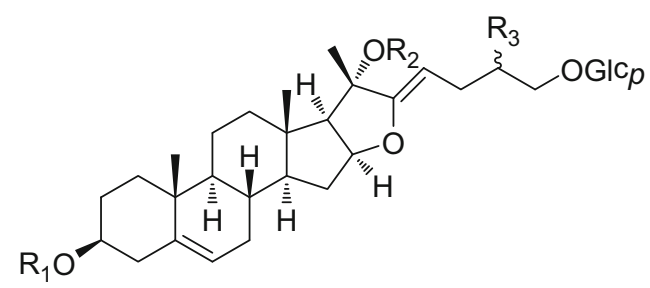

$$
\begin{aligned}
& 84 \mathrm{R}_{1}=\mathrm{S}_{9} \mathrm{R}_{2}=\mathrm{H} \quad \mathrm{R}_{3}=\alpha-\mathrm{CH}_{3} \\
& 85 R_{1}=S_{9} R_{2}=H \quad R_{3}=\beta-C_{3} \\
& 86 R_{1}=S_{13} R_{2}=H \quad R_{3}=\beta-C_{3} \\
& 87 R_{1}=S_{12} R_{2}=H \quad R_{3}=\beta-C_{3} \\
& 88 R_{1}=S_{9} R_{2}=C H_{3} R_{3}=\beta-C_{3}
\end{aligned}
$$

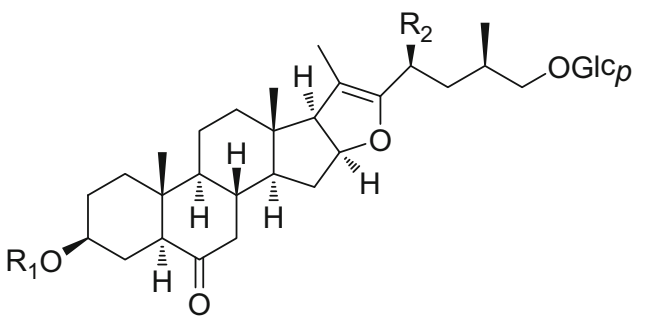

$$
\begin{aligned}
& 89 R_{1}=H \quad R_{2}=H \\
& 90 R_{1}=H \quad R_{2}=O H \\
& 91 R_{1}=S_{16} R_{2}=H
\end{aligned}
$$

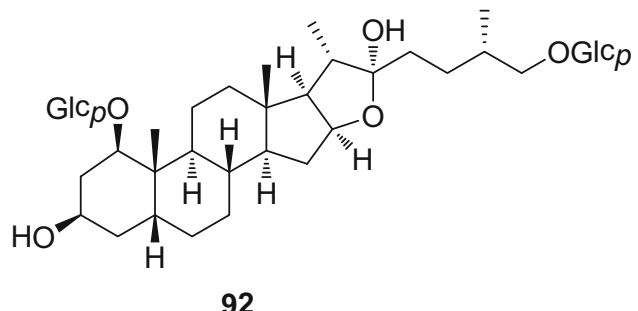

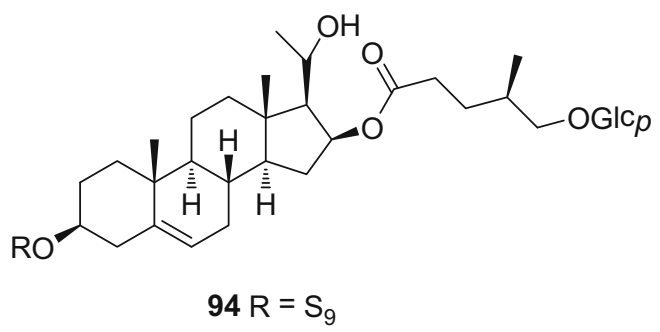

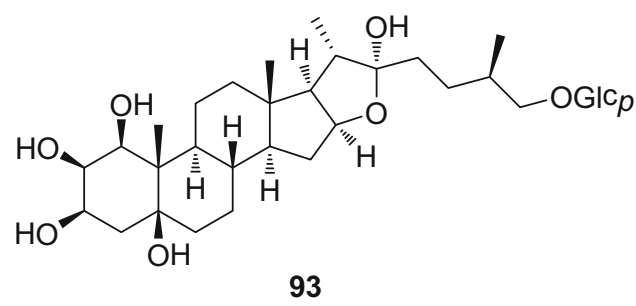

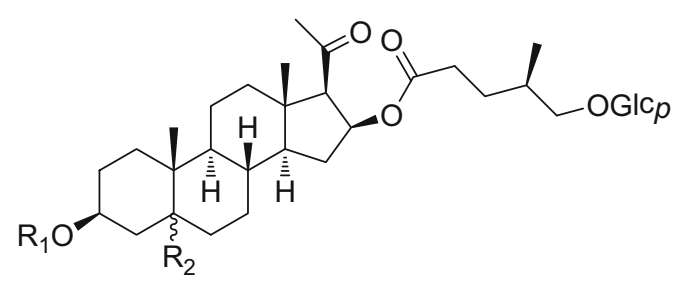

$$
\begin{array}{ll}
95 R_{1}=S_{23} & R_{2}=\alpha_{-H} \\
96 R_{1}=S_{25} & R_{2}=\alpha_{-H} \\
97 R_{1}=S_{25} & R_{2}=\beta-H
\end{array}
$$<smiles>[R6]C1CC[C@]2(C)C(=CC[C@H]3[C@H]4C[C@H](OCCCC(CO)CO)[C@@H](C(C)O)[C@@]4(C)CC[C@]32C)C1</smiles>

$98 \mathrm{R}=\mathrm{S}_{9}$

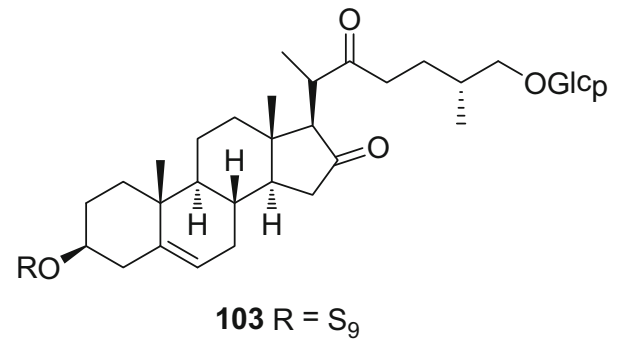

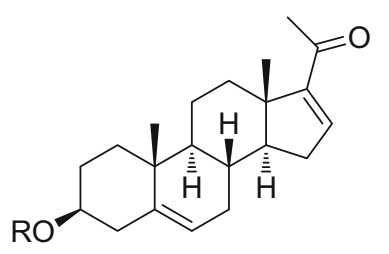

$99 \mathrm{R}=\mathrm{S}_{9}$ $100 \mathrm{R}=\mathrm{S}_{13}$ $101 \mathrm{R}=\mathrm{S}_{28}$

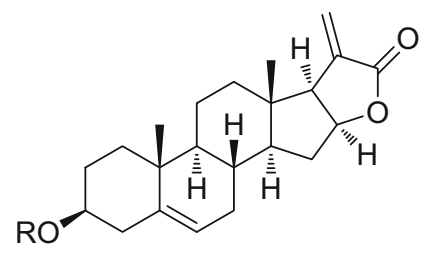

$102 \mathrm{R}=\mathrm{S}_{12}$

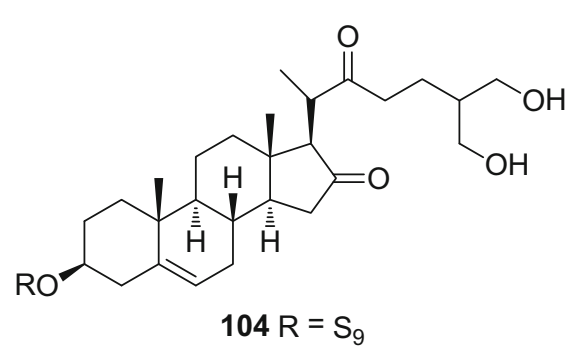

Fig. 5 Structures of compounds 84-104 


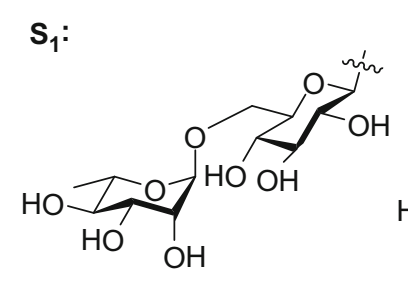

$S_{5}:$

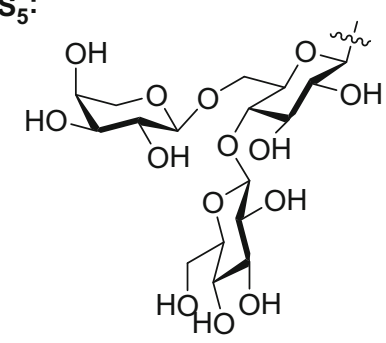

$\mathrm{S}_{8}:$

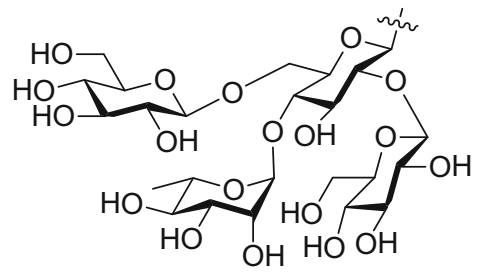

$\mathrm{S}_{2}:$

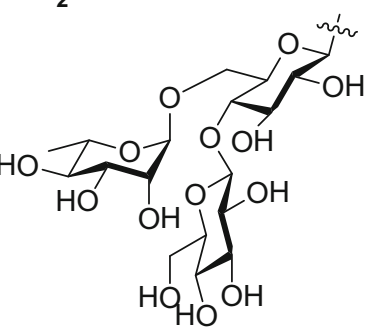

$\mathrm{S}_{3}:$

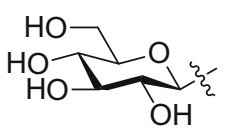

$\mathrm{S}_{4}:$<smiles>CC(C)OC(O)COC1(CO)OC(CO)C(O)C1O</smiles>

$\mathrm{s}_{7}:$

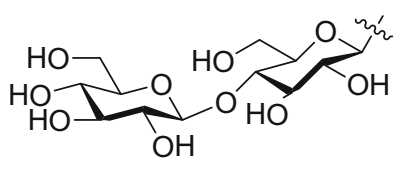

$\mathrm{HO}_{\mathrm{HOH}}$

$S_{9}:$

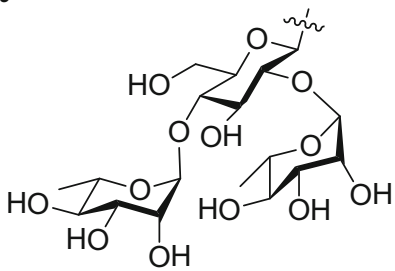

$S_{10}:$

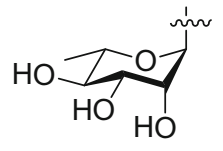

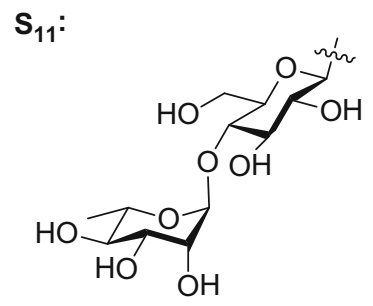

$S_{12}:$

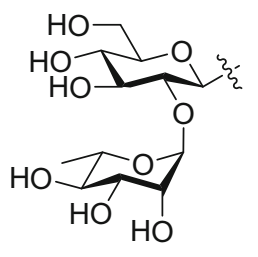

$\mathrm{S}_{13}:$

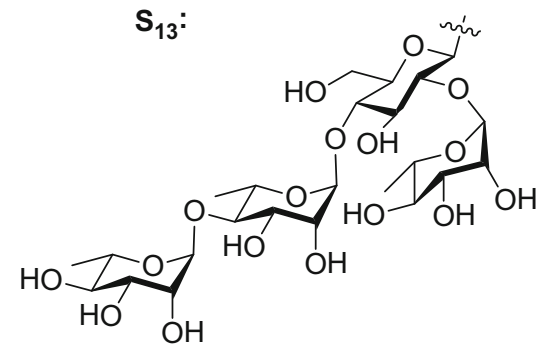

$\mathrm{S}_{14}$ :

$\mathrm{S}_{15}$ :

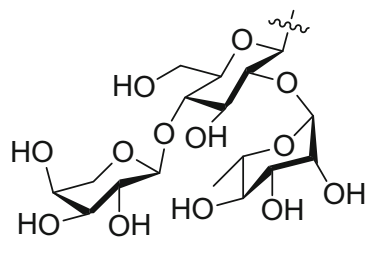

$$
\mathrm{S}_{16} \text { : }
$$

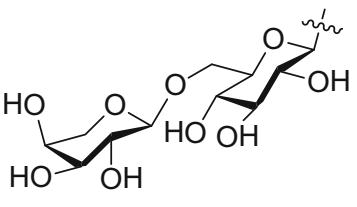

Fig. 6 Sugar residues of $S_{1}-S_{16}$

structure-activity relationships between the saponin structures and antifungal activities, the following points were suggested: (1) the close F ring is essential for the antifungal activities. (2) The cis/trans fusion between rings A and B has no significant difference in terms of antifungal activities. (3) Steroidal saponins bearing a saccharidic chain with more than one sugar were better antifungal agents (Figs. 6, 7). 


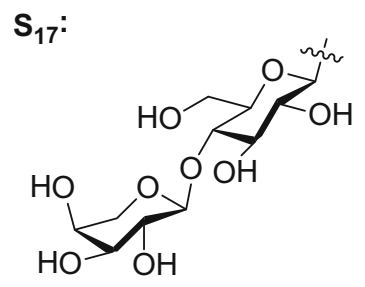

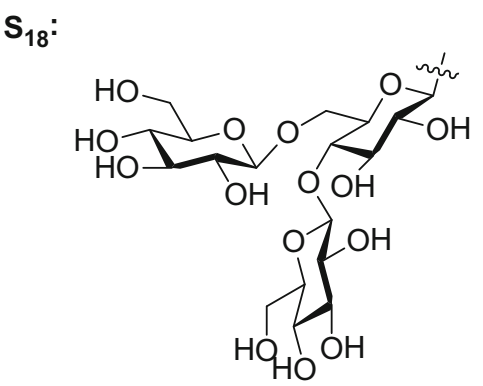

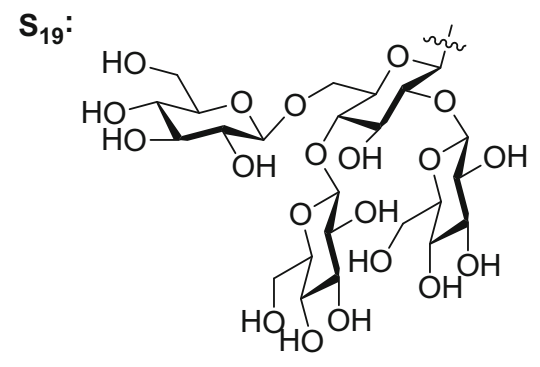

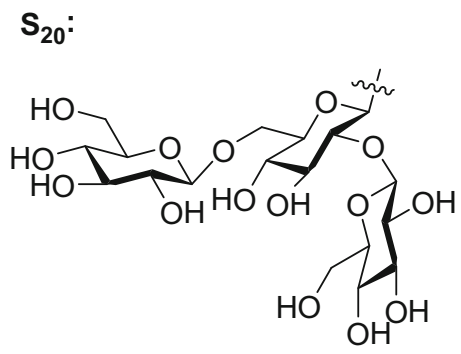

$\mathrm{S}_{21}:$

$\mathrm{S}_{22}$ :

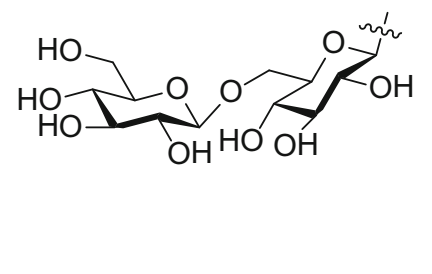

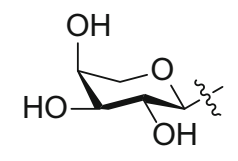

$S_{23}$ :

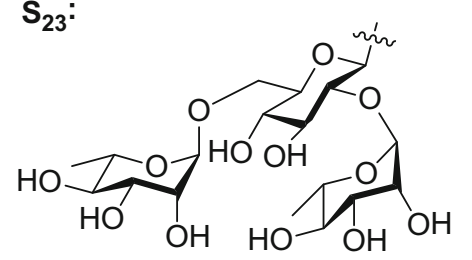

$\mathrm{S}_{24}:$

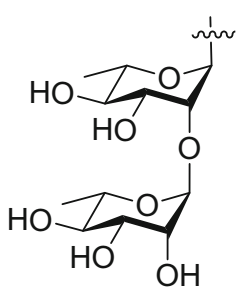

$\mathrm{S}_{26}$ :

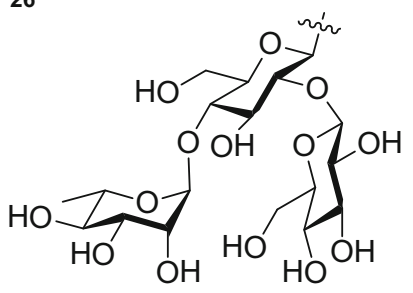

$\mathrm{S}_{29}:$

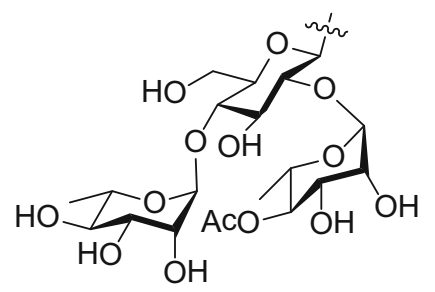

$S_{27}:$

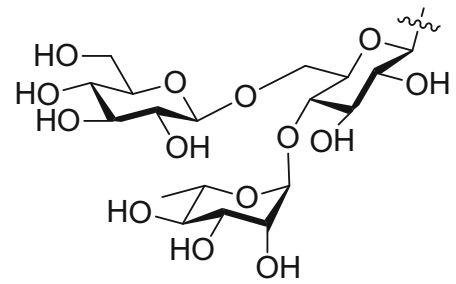

$\mathrm{S}_{30}:$

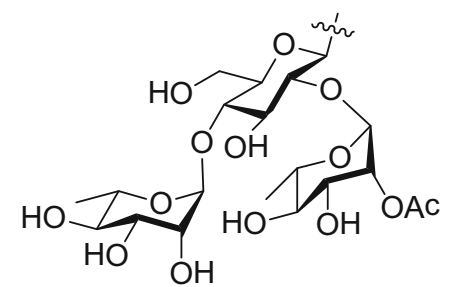

$\mathrm{S}_{25}$ :

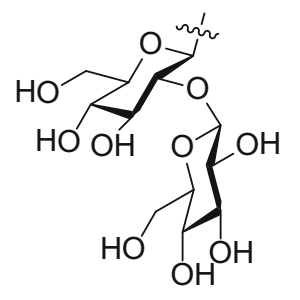

$\mathrm{S}_{28}:$

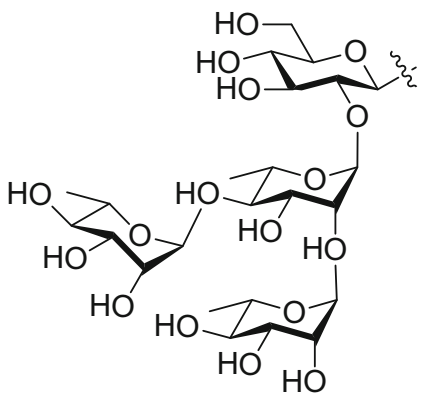

$\mathrm{S}_{31}$ :

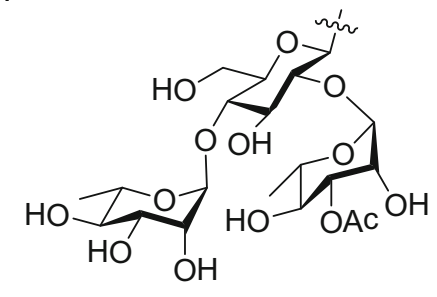

Fig. 7 Sugar residues of $S_{17}-S_{31}$

\subsection{Cytotoxicity}

Spirostane glycoside 9 and four furostane glycosides 79-82 were evaluated for their cytotoxicities against six human cancer cells (NFF, Hela, HT29, MCF7, MM96L, and K562). Compounds $\mathbf{7 9}$ and 80 selectively inhibited the proliferation of the HT29 colon cancer cell lines with $\mathrm{IC}_{50}$ values of 4.8 and $5.0 \mu \mathrm{g} / \mathrm{mL}$, respectively; while 
compounds 80 and $\mathbf{8 1}$ showed significant cytotoxicities aganist MCF7 cell lines with $\mathrm{IC}_{50}$ values of 9.5 and $3.4 \mu \mathrm{g} /$ $\mathrm{mL}$, respectively [11]. Compounds $\mathbf{2 4}, \mathbf{2 5}, \mathbf{2 8}, \mathbf{3 8}$, and $\mathbf{3 9}$, were evaluated for the cytotoxicities against three human cancer cell lines (A549, LAC and Hela). Only compound 38 possessed significant cytotoxicities with $\mathrm{IC}_{50}$ values of $3.70,5.70$ and $3.64 \mu \mathrm{M}$, respectively [21]. Another cytotoxic compound is isospirostane glycoside 32, which displayed potent cytotoxicities against the Hela and SMMC7221 cancer cell lines with $\mathrm{IC}_{50}$ values of $9.73 \pm 1.64$ and $21.54 \pm 1.64 \mu \mathrm{M}$, respectively [28]. The above results indicated that the hydroxyl substitutions on C-6 or C-17 of isospirostane glycosides decrease the cytotoxicities. Furostane glycoside 69 showed cytotoxicities against the Hela and SMMC-7221 cancer cell lines with $\mathrm{IC}_{50}$ values of $18.79 \pm 1.12$ and $28.57 \pm 1.57 \mu \mathrm{M}$, respectively; while the demethylated analogue 67 and the dehydrated analogues 89-91 showed no obvious cytotoxicities. Additionally, the sapogenin $\mathbf{7 0}$ was less cytotoxicities than that of corresponding glycoside 69 [28]. Compounds 11, 60, 85, $\mathbf{8 8}, 94,98$ and 103 , were tested for their cytotoxicities against SHSY5Y, SGC-7901, HCT-116 and Lovo cell lines. Only compound $\mathbf{9 8}$ showed significant cytotoxicities against SGC-7901 and HCT-116 cell lines with $\mathrm{IC}_{50}$ values of 8.1 and $5.5 \mu \mathrm{M}$, respectively [32].

\subsection{Anti-inflammatory Activity}

The aqueous extracts of the tubers of $S$. china showed the similar anti-inflammatory effects in vivo to that of acetylsalicylic acid (200 mg/kg, i.g.) [36]. Sieboldogenin (33) showed significant lipoxygenase inhibition activity with $\mathrm{IC}_{50}$ value of $38 \mu \mathrm{M}$. It also exhibited significant inhibition on carrageenan-induced hind paw oedema at the doses of 10 and $50 \mathrm{mg} / \mathrm{kg}$ [22]. Compounds 13, 14, 16, 48, 84-87, and 104 inhibited the lipoposaccharide (LPS) induced prostaglandin $\mathrm{E}_{2}\left(\mathrm{PGE}_{2}\right)$ production in murine peritoneal macrophages by $81.5,81.7,76.5,82.5,76.1,59.1,78.5$, 75.9 , and $82.0 \%$, respectively, at the concentration of $10 \mu \mathrm{M}$. These nine compounds also moderately inhibited the tumor necrosis factor $\alpha(\mathrm{TNF} \alpha)$ production on LPS stimulated murine peritoneal macrophages [30].

\section{Prospects}

The plants of the genus Smilax are widely spread in China. Their medical use for the treatment of inflammation and rheumatism has a long history in folk China. Previous studies on chemical constituents of Smilax sp. yielded diversified steroidal saponin. However, the biological activities studies of these isolated steroidal saponins lag behind, especially in anti-inflammatory related activities.
Acknowledgements This work was financially supported by the State Key Laboratory of Phytochemistry and Plant Resources in West China, Kunming Institute of Botany, Chinese Academy of Sciences (No. P2015-KF07), Science and Technology Program of Guangzhou, China (No. 201607010147), and Guangdong Medical Science Foundation (No. A2015225).

\section{Compliance with Ethical Standards}

Conflicts of interest The authors declare no conflict of interest.

Open Access This article is distributed under the terms of the Creative Commons Attribution 4.0 International License (http:// creativecommons.org/licenses/by/4.0/), which permits unrestricted use, distribution, and reproduction in any medium, provided you give appropriate credit to the original author(s) and the source, provide a link to the Creative Commons license, and indicate if changes were made.

\section{References}

1. Editorial Committee of the flora of the Chinese Aacdemy of Sciences, 'Flora Reipublicae Popularis Sinicae', Beijing House of Science Press (1978)

2. Chinese Pharmacopoeia Commission, Chinese Pharmacopoeia (China Medical Science Press, Beijing, 2015)

3. Compilation Group of Chinese Hebral Medicine, The Assembly of Chinese Herbal Medicine (People's Medical Publishing House, Beijing, 1975)

4. H. Ma, W. Wang, F. Dong, Zhongguo Yaofang 20, 1191-1193 (2009)

5. Y. Yi, Y. Liu, Y. Peng, Y. Xie, S. Wang, Zhongguo Yaofang 25, 1430-1432 (2011)

6. J. Guo, F. Qian, J. Li, Q. Xu, T. Chen, Clin. Chem. 53, 465-471 (2007)

7. R.R. Bernardo, A.V. Pinto, J.P. Parente, Phytochemistry 43, 465-469 (1996)

8. Y. Sashida, S. Kubo, Y. Mimaki, T. Nikaido, T. Ohmoto, Phytochemistry 31, 2439-2443 (1992)

9. K.Y. Cho, Yakhak Hoechi 39, 141-147 (1995)

10. Z. Belhouchet, M. Sautour, T. Miyamoto, M.A. Lacaille-Dubois, Chem. Pharm. Bull. 56, 1324-1327 (2008)

11. V.L. Challinor, P.G. Parsons, S. Chap, E.F. White, J.T. Blanchfield, R.P. Lehmann, J.J. De Voss, Steroids 77, 504-511 (2012)

12. G.B. Iskenderov, M.N. Mamedova, N.I. Musaev, Chem. Nat. Comp. 11, 820 (1975)

13. Y. Ju, Z.J. Jia, Phytochemistry 31, 1349-1351 (1992)

14. Y. Ju, Z.J. Jia, Phytochemistry 33, 1193-1195 (1993)

15. Y. Ju, H.R. Peng, Z.J. Jia, X.J. Sun, J. Lanzhou Univ. (Nat. Sci.) 30, 64-67 (1994)

16. X.J. Sun, Y. Ju, M. Du, Z.J. Jia, Zhongcaoyao 26, 395-396 (1995)

17. A. Ivanova, B. Mikhova, I. Klaiber, D. Dinchev, I. Kostova, Nat. Prod. Res. 23, 916-924 (2009)

18. T. Lin, H.L. Huang, R.H. Liu, J.C. Shu, G. Ren, F. Shao, L.S. Liu, Magn. Reson. Chem. 50, 813-817 (2012)

19. J. Xu, X. Li, C.C. Zhao, Y. Wang, Nat. Prod. Res. 22, 884-889 (2008)

20. X.H. Wu, C.Z. Wang, S.Q. Wang, C. Mi, Y. He, J. Zhang, Y.W. Zhang, S. Anderson, C.S. Yuan, J. Ethnopharmacol. 162, 362-368 (2015)

21. C. Zhang, S. Feng, L. Zhang, Z. Ren, Nat. Prod. Res. 27, 1255-1260 (2013) 
22. I. Khan, M. Nisar, F. Ebad, S. Nadeem, M. Saeed, H. Khan, F. Samiullah, N.Karim Khuda, Z. Ahamd, J. Ethnopharmacol. 121, 175-177 (2009)

23. S. Kubo, Y. Mimaki, Y. Sashida, T. Nikaido, T. Ohmoto, Phytochemistry 31, 2445-2450 (1992)

24. P.Y. Hou, C. Mi, Y. He, J. Zhang, S.Q. Wang, F. Yu, S. Anderson, Y.W. Zhang, X.H. Wu, Fitoterapia 105, 43-48 (2015)

25. M. Sautour, T. Miyamoto, M.A. Lacaille-Dubois, J. Nat. Prod. 68, 1489-1493 (2005)

26. M. Sautour, T. Miyamoto, M.A. Lacaille-Dubois, Planta Med. 72, 667-670 (2006)

27. H.L. Huang, R.H. Liu, F. Shao, Magn. Reson. Chem. 47, 741-745 (2009)

28. J. Xu, S. Feng, Q. Wang, Y. Cao, M. Sun, C. Zhang, Molecules 19, 20975-20987 (2014)

29. B.P. da Silva, J.P. Parente, Z. Naturforsch. B 63, 95-100 (2008)

30. B. Shao, H. Guo, Y. Cui, M. Ye, J. Han, D. Guo, Phytochemistry 68, 623-630 (2007)

31. A. Ivanova, B. Mikhova, T. Batsalova, B. Dzhambazov, I. Kostova, Fitoterapia 82, 282-287 (2011)

32. F. Liang, J.W. He, G.H. Zhu, R.H. Liu, J.C. Shu, F. Shao, H.L. Huang, Phytochem. Lett. 16, 294-298 (2016)

33. Y. Ju, Z. Jia, X. Sun, Phytochemistry 37, 1433-1436 (1994)

34. V.L. Challinor, J.J. De Voss, Nat. Prod. Rep. 30, 429-454 (2013)

35. C.R. Yang, Y. Zhang, M.R. Jacob, S.I. Khan, Y.J. Zhang, X.C. Li, Antimicrb. Agents. Chemother. 50, 1710-1714 (2006)

36. X.S. Shu, Z.H. Gao, X.L. Yang, J. Ethnopharmacol. 103, 327-332 (2006)

37. W.S. Laitonjam, B.D. Kongbrailatpam, Nat. Prod. Res. 24, 1168-1176 (2010)
38. R. Tschesche, G. Lüdke, G. Wulff, Tetrahedron Lett. 8, 2785-2790 (1967)

39. Y. Ju, Z.J. Jia, H.R. Peng, X.J. Sun, Chin. Chem. Lett. 4, 137-138 (1993)

40. Y. Ju, Z.J. Jia, Chem. J. Chin. Univ. 11, 1386-1387 (1990)

41. Z.H. Jia, Y. Ju, M. Du, Chin. Chem. Lett. 3, 431-432 (1992)

42. Y. Ju, Z.J. Jia, Y.N. Wu, Chin. Chem. Lett. 2, 853-854 (1991)

43. C.L. Zhang, W. Zhu, Y.M. Cheng, W.C. Li, Xibei Zhiwu Xuebao 23, 1973-1976 (2003)

44. C.L. Zhang, J.M. Gao, W. Zhu, Phytochem. Lett. 5, 49-52 (2012)

45. M.H. Woo, J.C. Do, K.H. Son, J. Nat. Prod. 55, 1129-1135 (1992)

46. C.L. Zhang, W. Zhu, X.M. Li, B.F. Su, X.Y. Yan, Linye Kexue 42, 69-73 (2006)

47. Z.H. Jia, Y. Ju, Phytochemistry 31, 3173-3175 (1992)

48. C.L. Zhang, W.C. Li, J.M. Gao, J.X. Fu, J. Northwest Sci-Tech Univ. of Agric. For. (Nat. Sci.) 31, 163-166 (2003)

49. Y. Ju, Z.J. Jia, Chem. J. Chin. Univ. 12, 1488-1489 (1991)

50. C. Lavaud, Planta Med. Phytother. 26, 64-73 (1993)

51. J.Y. Liu, J.X. Fu, J.M. Gao, M.H. Qiu, J. Northwest Sci-Tech Univ. of Agric. For. (Nat. Sci.) 30, 222-224 (2002)

52. W. Chen, X.A. Shou, Y. Chen, N. Qin, W. Qiao, S.A. Tang, H.Q. Duan, Chem. Nat. Comp. 50, 989-993 (2014)

53. J. Li, X. Bi, G. Zheng, Y. Hitoshi, T. Ikeda, T. Nohara, Chem. Pharm. Bull. 54, 1451-1454 (2006)

54. X.H. Wu, C.Z. Wang, J. Zhang, S.Q. Wang, L. Han, Y.W. Zhang, C.S. Yuan, Phytother. Res. 28, 1822-1828 (2014)

55. X.H. Wu, J. Zhang, S.Q. Wang, V.C. Yang, S. Anderson, Y.W. Zhang, Phytomedicine 21, 1196-1201 (2014) 